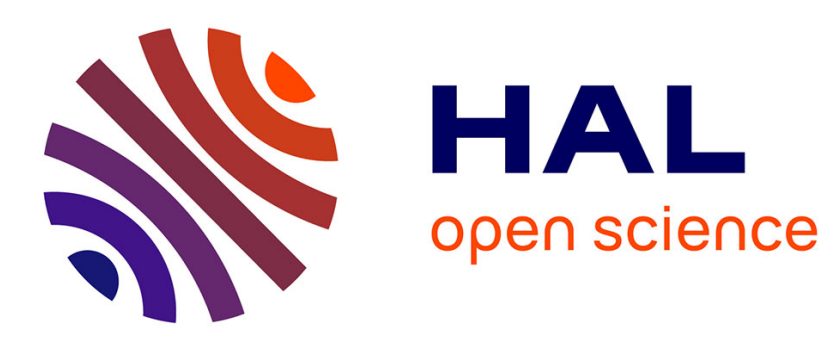

\title{
Aggregation functions: construction methods, conjunctive, disjunctive and mixed classes
}

Michel Grabisch, Jean-Luc Marichal, Radko Mesiar, Endre Pap

\section{To cite this version:}

Michel Grabisch, Jean-Luc Marichal, Radko Mesiar, Endre Pap. Aggregation functions: construction methods, conjunctive, disjunctive and mixed classes. Information Sciences, 2011, 181 (1), pp.23-43. 10.1016/j.ins.2010.08.040 . hal-00539032

\section{HAL Id: hal-00539032 \\ https://hal.science/hal-00539032}

Submitted on 5 Jan 2011

HAL is a multi-disciplinary open access archive for the deposit and dissemination of scientific research documents, whether they are published or not. The documents may come from teaching and research institutions in France or abroad, or from public or private research centers.
L'archive ouverte pluridisciplinaire HAL, est destinée au dépôt et à la diffusion de documents scientifiques de niveau recherche, publiés ou non, émanant des établissements d'enseignement et de recherche français ou étrangers, des laboratoires publics ou privés. 


\title{
Aggregation functions: construction methods, conjunctive, disjunctive and mixed classes
}

\author{
Michel GRABISCH \\ University of Panthéon-Sorbonne \\ Paris, France \\ Jean-Luc MARICHAL \\ University of Luxembourg \\ Luxembourg \\ Radko MESIAR \\ Slovak University of Technology \\ Bratislava, Slovakia, \\ Endre PAP \\ University of Novi Sad \\ Novi Sad, Serbia
}

December 19, 2010

\begin{abstract}
In this second part of our state-of-the-art overview on aggregation theory, based again on our recent monograph on aggregation functions, we focus on several construction methods for aggregation functions and on special classes of aggregation functions, covering the well-known conjunctive, disjunctive, and mixed aggregation functions. Some fields of applications are included.
\end{abstract}

\section{Introduction}

This paper is a continuation of [29] and it is based on our monograph [28], as explained in the first part of this paper, i.e., in [29]. This two-part overview paper is an invited state-of-art overview for Information Sciences, and as all necessary details are in [28] (as mentioned in introduction), we do not give here proofs, detailed related references etc. Using the same notation and terminology as in [29], this paper is organized as follows. The next section deals with construction methods for aggregation functions. Section 3 is devoted to conjunctive aggregation functions, with a special stress on distinguished classes of triangular norms and copulas. In Section 4, disjunctive aggregation functions are discussed, though due to their duality with conjunctive aggregation functions, many results are omitted. Mixed aggregation functions, mixing in some sense conjunctive and disjunctive aggregation functions, are introduced in Section 5. In concluding remarks, several applied fields where aggregation functions play an important role are summarized. 


\section{Some construction methods for aggregation functions}

There is a strong demand for an ample variety of aggregation functions having predictable and tailored properties to be used in modelling processes. Several construction methods have been introduced and developed for extending the known classes of aggregation functions (defined either on $[0,1]$ or, possibly, on some other domains). In this paper we present some well-established construction methods as well as some new ones.

The first group of construction methods can be characterized "from simple to complex". They are based on standard arithmetical operations on the real line and fixed real functions. The second group of construction methods starts from given aggregation functions to construct new ones. Here we can start either from aggregation functions with a fixed number of inputs (e.g., from binary functions only) or from extended aggregation functions which have multiple arities. Observe that some methods presented are applicable to all aggregation functions (for example, transformation), while some of them can be applied only to specific cases. Finally, there are construction methods allowing us to find aggregation functions when only some partial knowledge about them is available. For more details on this topic we recommend Chapter 6 in [28].

\subsection{Transformation of aggregation functions}

The idea of transformation of aggregation functions can be clearly illustrated on the well-known relation of the two basic arithmetic operations of addition and multiplication

$$
\sum_{i=1}^{n}\left(-\log x_{i}\right)=-\log \prod_{i=1}^{n} x_{i} .
$$

Formally, (1) can be rewritten as

$$
\prod_{i=1}^{n} x_{i}=\varphi^{-1}\left(\sum_{i=1}^{n} \varphi\left(x_{i}\right)\right)
$$

where $\varphi:] 0, \infty[\rightarrow]-\infty, \infty[$ is given by $\varphi(x)=-\log x$.

Proposition 1 Let $\mathbb{I}, \mathbb{J}$ be real intervals and let $\varphi: \mathbb{J} \rightarrow \mathbb{I}$ be a monotone bijection. For $n \in \mathbb{N}$, let $\mathrm{A}: \mathbb{I}^{n} \rightarrow \mathbb{I}$ be an n-ary aggregation function. Then the function $\mathrm{A}_{\varphi}: \mathbb{J}^{n} \rightarrow \mathbb{J}$ given by

$$
\mathrm{A}_{\varphi}\left(x_{1}, \ldots, x_{n}\right):=\varphi^{-1}\left(\mathrm{~A}\left(\varphi\left(x_{1}\right), \ldots, \varphi\left(x_{n}\right)\right)\right)
$$

is an n-ary aggregation function on $\mathbb{J}^{n}$.

Observe that the transformed aggregation functions $\mathrm{A}_{\varphi}$ inherit the algebraic and topological properties of the original aggregation function $\mathrm{A}$ (and then $\varphi$ is an isomorphism between $(\mathbb{I}, A)$ and $\left(\mathbb{J}, A_{\varphi}\right)$ ), for example the associativity, symmetry, bisymmetry, existence of the neutral element and/or annihilator, continuity, strict monotonicity, etc. On the other hand, analytical properties of $A$ need not be inherited by the transform $A_{\varphi}$, in general. This is, for example, the case of the Lipschitz property, difference scale invariance, homogeneity, 
additivity, linearity, etc. Transformations can be applied consecutively, i.e., if A $: \mathbb{I}^{n} \rightarrow \mathbb{I}$ is a given aggregation function, and $\varphi: \mathbb{J} \rightarrow \mathbb{I}$ and $\psi: \mathbb{L} \rightarrow \mathbb{J}$ are given monotone bijections, then $\mathrm{A}_{\varphi}$ is an aggregation function on $\mathbb{J}$ and hence $\left(\mathrm{A}_{\varphi}\right)_{\psi}$ is an aggregation function on $\mathbb{L}$. We have

$$
\left(\mathrm{A}_{\varphi}\right)_{\psi}=\mathrm{A}_{\varphi \circ \psi} .
$$

Note that quasi-arithmetic means, see Section 3.1 in [29], can be seen as transformations of the standard arithmetic mean. Similarly, strict t-norms (see Section 3) and representable uninorms (see Section 5) are related to the standard addition.

Transformations of aggregation functions on $\mathbb{I}^{n}$ into aggregation function on $\mathbb{J}^{n}$ by means of a monotone bijection $\varphi: \mathbb{J} \rightarrow \mathbb{I}$ can be seen as a change of the scale (recall the logarithmic scale). In the case $\mathbb{I}=\mathbb{J}$ one can discuss the invariance of an aggregation function $\mathrm{A}$ under $\varphi$-transform, $\mathrm{A}=\mathrm{A}_{\varphi}$. As a typical example recall the transformation $\varphi:[0, \infty[\rightarrow[0, \infty[$ given by $\varphi(x)=$ $c x$, where $c$ is a fixed positive constant. Then the summation $\Sigma:[0, \infty]^{n} \rightarrow$ $[0, \infty]$ remains unchanged under $\varphi$-transform, $\Sigma_{\varphi}=\Sigma$. As an example of an aggregation function on any closed or open interval $\mathbb{I}^{n}$ which is invariant under each monotone bijection $\varphi: \mathbb{I} \rightarrow \mathbb{I}$, consider the function $\mathrm{A}:[0,1]^{4} \rightarrow[0,1]$ given by

$$
\mathrm{A}(\mathbf{x})=\operatorname{Med}\left(\operatorname{Med}\left(x_{1}, x_{2}, x_{3}\right), x_{2}, x_{4}\right) .
$$

For more details we recommend Section 6.2 and Chapter 7 in [28].

\subsection{Composed aggregation}

The idea of composed aggregation has two different roots leading to two different composition construction methods. The first is linked to the notion of associativity.

Proposition 2 Let $\mathbb{I}$ be a real interval, $n, m \in \mathbb{N}$, and let $\mathrm{A}: \mathbb{I}^{2} \rightarrow \mathbb{I}, \mathrm{B}: \mathbb{I}^{n} \rightarrow \mathbb{I}$ and $\mathrm{C}: \mathbb{I}^{m} \rightarrow \mathbb{I}$ be aggregation functions. Then the composite function $\mathrm{D}_{\mathrm{A} ; \mathrm{B}, \mathrm{C}}$ : $\mathbb{I}^{n+m} \rightarrow \mathbb{I}$ given by

$$
\mathrm{D}_{\mathrm{A} ; \mathrm{B}, \mathrm{C}}\left(x_{1}, \ldots, x_{n+m}\right):=\mathrm{A}\left(\mathrm{B}\left(x_{1}, \ldots, x_{n}\right), \mathrm{C}\left(x_{n+1}, \ldots, x_{n+m}\right)\right)
$$

is an aggregation function on $\mathbb{I}^{n+m}$.

Remark 1 i) Construction (5) can be generalized by induction to the case of a $k$-ary outer aggregation function $\mathrm{A}$ and $k$ inner aggregation functions $\mathrm{B}_{1}, \ldots, \mathrm{B}_{k}$ defined on $n_{1-}, \ldots, n_{k}$-dimensional input vectors, i.e., for $n=$ $\sum_{i=1}^{k} n_{i}$ we obtain an aggregation function $\mathrm{D}_{\mathrm{A}_{;} \mathrm{B}_{1}, \ldots, \mathrm{B}_{k}}: \mathbb{I}^{n} \rightarrow \mathbb{I}$ given by

$$
\mathrm{D}_{\mathrm{A} ; \mathrm{B}_{1}, \ldots, B_{k}}\left(\mathrm{x}_{1}, \ldots, \mathbf{x}_{k}\right):=\mathrm{A}\left(\mathrm{B}_{1}\left(\mathbf{x}_{1}\right), \ldots, \mathrm{B}_{k}\left(\mathbf{x}_{k}\right)\right),
$$

where $\mathbf{x}_{i} \in \mathbb{I}^{n_{i}}, i \in[k]$.

ii) Construction (5) is also known as double aggregation, and was deeply investigated in [14].

The second root of composition-based constructions of aggregation functions comes from the standard composition of real functions. 
Proposition 3 Let $\mathbb{I}$ be a real interval and let $k, n \in \mathbb{N} \backslash\{1\}$. Let $\mathrm{A}: \mathbb{I}^{k} \rightarrow \mathbb{I}$ and $\mathrm{B}_{1}, \ldots, \mathrm{B}_{k}: \mathbb{I}^{n} \rightarrow \mathbb{I}$ be aggregation functions. Then the function $\mathrm{C}=$ $\mathrm{A}\left(\mathrm{B}_{1}, \ldots, \mathrm{B}_{k}\right): \mathbb{I}^{n} \rightarrow \mathbb{I}$ given by

$$
\mathrm{C}(\mathrm{x}):=\mathrm{A}\left(\mathrm{B}_{1}(\mathbf{x}), \ldots, \mathrm{B}_{k}(\mathbf{x})\right)
$$

is an aggregation function on $\mathbb{I}^{n}$.

Example 1 Let $\mathrm{A}$ in (6) be a weighted arithmetic mean with weights $w_{1}, \ldots, w_{k}$. Then the function $\mathrm{C}$ given by (6) is the well-known convex combination (convex sum) of aggregation functions $\mathrm{B}_{1}, \ldots, \mathrm{B}_{k}$,

$$
\mathrm{C}=\sum_{i=1}^{k} w_{i} \mathrm{~B}_{i}
$$

Several special classes of aggregation functions can be obtained by the construction (6), such as the ( $n$-ary with $n>2)$ uninorms or nullnorms discussed in Section 5.

An essential difference between composition-based constructions (5) and (6) is in the formal occurrence of single inputs $x_{i}$ in the composed aggregation. Indeed, in (5) each input $x_{i}$ is aggregated by exactly one inner function $\mathrm{B}$ or $\mathrm{C}$ (in the extended version described in Remark 1 (i) by exactly one $\mathrm{B}_{j}$ ). On the other hand, in the case (6), each input $x_{i}$ is aggregated by any of the involved inner aggregation functions $\mathrm{B}_{1}, \ldots, \mathrm{B}_{k}$. Several intermediate approaches can be introduced, too.

\subsection{Weighted aggregation functions}

Let $m_{1}, \ldots, m_{n} \in \mathbb{N}_{0}$, such that at least one $m_{1}, \ldots, m_{n}$ is positive, be given integer weights and let $\mathbf{m}:=\left(m_{1}, \ldots, m_{n}\right)$ be the corresponding nonzero weight vector. For a given symmetric extended aggregation function $A: \cup_{n \in \mathbb{N}} \mathbb{I}^{n} \rightarrow \mathbb{I}$, we can incorporate weights $m_{i}$ as follows:

$$
\mathrm{A}_{\mathbf{m}}(\mathbf{x}):=\mathrm{A}\left(m_{1} \cdot x_{1}, m_{2} \cdot x_{2}, \ldots, m_{n} \cdot x_{n}\right),
$$

where $m_{i} \cdot x_{i}=x_{i}, \ldots, x_{i}$ ( $m_{i}$ times). Applying (7) to the arithmetic mean AM, we obtain the class of weighted means (with rational weights $m_{i} / \sum_{j=1}^{n} m_{j}$ ).

The above cardinality-based approach of introducing weights into symmetric extended aggregation functions can be straightforwardly extended to rational weights in the case when the original extended aggregation function is not only symmetric but also strongly idempotent, i.e., for any $k \in \mathbb{N}$ and for any $\mathbf{x} \in$ $\cup_{n \in \mathbb{N}} \mathbb{I}^{n}$ we have $\mathrm{A}(k \cdot \mathbf{x})=\mathrm{A}(\mathbf{x})$, where $k \cdot \mathbf{x}$ represents the ordered list of the $(k \times n)$ values obtained by concatenating $k$ copies of $\mathbf{x}$ and then delating the parentheses. For general weights details can be found in [13].

Proposition 4 Let $\mathrm{A}: \cup_{n \in \mathbb{N}} \mathbb{I}^{n} \rightarrow \mathbb{I}$ be a symmetric strongly idempotent extended aggregation function, and let $\mathbf{v} \in\left[0, \infty\left[^{n}\right.\right.$ be an $n$-dimensional weight vector. For $k \in \mathbb{N}$, define $\mathbf{w}^{(k)}=\left(w_{1}^{(k)}, \ldots, w_{n}^{(k)}\right)$ by

$$
v_{i}^{(k)}:=\inf \left\{\frac{m}{k} \mid m \in \mathbb{N}_{0}, \frac{m}{k} \geqslant v_{i}\right\}, \quad \mathbf{w}^{(k)}:=\frac{\mathbf{v}^{(k)}}{\sum_{i=1}^{n} v_{i}^{(k)}} .
$$


Then $\mathbf{w}^{(k)}$ is a normalized rational weight vector for all $k \in \mathbb{N}$. Define a function $\mathrm{A}_{\mathbf{v}}: \mathbb{I}^{n} \rightarrow \mathbb{I}$ by

$$
A_{\mathbf{v}}(\mathbf{x}):=\liminf _{k \rightarrow \infty} A_{\mathbf{w}^{(k)}}(\mathbf{x}) .
$$

Then $\mathrm{A}_{\mathbf{v}}$ is an idempotent aggregation function on $\mathbb{I}^{n}$, which is called a (v)weighted aggregation function $\mathrm{A}$.

Example 2 (i) Let A : $\cup_{n \in \mathbb{N}} \mathbb{I}^{n} \rightarrow \mathbb{I}$ be a quasi-arithmetic mean, i.e., there exists a continuous strictly monotone function $f: \mathbb{I} \rightarrow[-\infty, \infty]$ such that

$$
\mathrm{A}(\mathbf{x})=f^{-1}\left(\sum_{i=1}^{n} \frac{f\left(x_{i}\right)}{n}\right)
$$

Applying Proposition 4, for any weight vector $\mathbf{v}$ we have

$$
\mathrm{A}_{\mathbf{v}}(\mathbf{x})=f^{-1}\left(\frac{\sum_{i=1}^{n} v_{i} f\left(x_{i}\right)}{\sum_{i=1}^{n} v_{i}}\right)=f^{-1}\left(\sum_{i=1}^{n} w_{i} f\left(x_{i}\right)\right)=\mathrm{A}_{\mathbf{w}}(\mathbf{x}),
$$

where

$$
\mathbf{w}=\frac{\mathbf{v}}{\sum_{i=1}^{n} v_{i}} .
$$

Hence $\mathrm{A}_{\mathbf{v}}=\mathrm{A}_{\mathbf{w}}$ is a (w-)weighted quasi-arithmetic mean.

(ii) Let $q:[0,1] \rightarrow[0,1]$ be a nondecreasing continuous function satisfying $q(0)=0$ and $q(1)=1$. For an arbitrary real interval $\mathbb{I}$, the mapping $\mathrm{OWA}_{q}: \cup_{n \in \mathbb{N}} \mathbb{I}^{n} \rightarrow \mathbb{I}$ given by

$$
\operatorname{OWA}_{q}(\mathbf{x}):=\sum_{i=1}^{n}\left(q\left(\frac{i}{n}\right)-q\left(\frac{i-1}{n}\right)\right) x_{(i)}
$$

where $\left(x_{(1)}, \ldots, x_{(n)}\right)$ is a nondecreasing permutation of $\left(x_{1}, \ldots, x_{n}\right)$, is an extended ordered weighted averaging function. Though $\mathrm{OWA}_{q}$ is not decomposable, in general, it is symmetric and strongly idempotent, and thus we can apply Proposition 4. For any normalized weight vector $\mathbf{w} \in$ $[0,1]^{n}$, we then have

$$
\mathrm{OWA}_{q, \mathbf{w}}(\mathbf{x})=\sum_{i=1}^{n}\left(q\left(c_{i}\right)-q\left(c_{i-1}\right)\right) x_{(i)},
$$

where $c_{i}:=\sum_{j=1}^{i} w_{j}$.

The approach to weighted aggregation as introduced in Proposition 4 can be understood as a quantitative approach (weights include the number of repetitions of inputs to be aggregated). In the case of an extended aggregation function $\mathrm{A}$ with an extended neutral element $e$, we can apply another approach which can be called qualitative (weights include how the data should be transformed before aggregation). Its idea is based on transformation of an input $x_{i}$ and the weight $u_{i}$ into a new quantity $y_{i}=h\left(u_{i}, x_{i}\right)$ and then to aggregate these new quantities $y_{i}$ by means of $A$. For deeper motivation, applications and some details on this approach (on the unit interval only, and under the name RET-operators) we recommend $[41,71]$. In this qualitative approach we deal with weight vectors $\mathbf{u} \in[0,1]^{n}$ such that $\operatorname{Max}(\mathbf{u})=1$. 
Definition 1 Let $\mathbb{I}$ be a real interval and $e \in \mathbb{I}$ a fixed constant. Let a function $h:[0,1] \times \mathbb{I} \rightarrow \mathbb{I}$ satisfy the following conditions:

(i) $h(0, x)=e$ and $h(1, x)=x$;

(ii) $h$ is nondecreasing in the second coordinate;

(iii) $h(\cdot, x)$ is nondecreasing for all $x \geqslant e$ and nonincreasing for all $x \leqslant e$.

Then $h$ is called an e-weighting transformation function.

Proposition 5 Let $\mathrm{A}: \cup_{n \in \mathbb{N}} \mathbb{I}^{n} \rightarrow \mathbb{I}$ be an extended aggregation function with an extended neutral element $e$. Let $h:[0,1] \times \mathbb{I} \rightarrow \mathbb{I}$ be an e-weighting transformation function, and let $\mathbf{u} \in[0,1]^{n}$ be a weight vector such that $\operatorname{Max}(\mathbf{u})=1$. Then the function $\mathrm{A}_{h, \mathbf{u}}: \cup_{n \in \mathbb{N}} \mathbb{I}^{n} \rightarrow \mathbb{I}$ given by

$$
\mathrm{A}_{h, \mathbf{u}}(\mathbf{x}):=\mathrm{A}\left(h\left(u_{1}, x_{1}\right), \ldots, h\left(u_{n}, x_{n}\right)\right)
$$

is an extended aggregation function.

Example 3 Each (binary) t-norm $\mathrm{T}$ is a 0 -weighting transformation function on the unit interval $[0,1]$, see Section 3.1. Recall that each t-conorm $\mathrm{S}$ is an extended aggregation function with extended neutral element 0 , see Section 4.Then for each $n$-dimensional $\mathbf{u}$ such that $\operatorname{Max}(\mathbf{u})=1$, the corresponding function $\mathrm{S}_{\mathbf{T}, \mathbf{u}}:[0,1]^{n} \rightarrow[0,1]$ is given by

$$
\mathrm{S}_{\mathrm{T}, \mathbf{u}}(\mathbf{x})=\mathrm{S}\left(\mathrm{T}\left(u_{1}, x_{1}\right), \ldots, \mathrm{T}\left(u_{n}, x_{n}\right)\right) .
$$

In the special case $\mathrm{T}=\operatorname{Min}$ and $\mathrm{S}=\operatorname{Max}$ we obtain the well-known weighted maximum, given by

$$
\operatorname{Max}_{\operatorname{Min}, \mathbf{u}}(\mathbf{x})=\operatorname{Max}\left(\operatorname{Min}\left(u_{1}, x_{1}\right), \ldots, \operatorname{Min}\left(u_{n}, x_{n}\right)\right) .
$$

\subsection{Aggregation functions based on minimal dissimilarity}

For the Euclidean metric $d$ on $\mathbb{R}^{n}\left(L_{2}\right.$ metric),

$$
d(\mathbf{x}, \mathbf{y}):=\sqrt{\sum_{i=1}^{n}\left(x_{i}-y_{i}\right)^{2}},
$$

it is well known that for each $\mathbf{x} \in \mathbb{R}^{n}$ there exists a unique $s \in \mathbb{R}$ such that

$$
d(\mathbf{x}, n \cdot s)=\inf \{d(\mathbf{x}, n \cdot r) \mid r \in \mathbb{R}\},
$$

and that $s=\mathrm{AM}(\mathbf{x})$. Hence the arithmetic mean can be understood as the solution of a minimization problem (projection, in fact). Similarly, the Chebyshev metric $c$ on $\mathbb{R}^{n}$ ( $L_{\infty}$ metric),

$$
c(\mathbf{x}, \mathbf{y}):=\|\mathbf{x}-\mathbf{y}\|_{\infty},
$$

yields the arithmetic mean of Min and Max,

$$
s=\frac{\operatorname{Min}(\mathbf{x})+\operatorname{Max}(\mathbf{x})}{2}
$$


(observe that this is an ordered weighted averaging function). On the other hand, for the "Manhattan distance" ( $L_{1}$ metric) $l$ on $\mathbb{R}^{n}$,

$$
l(\mathbf{x}, \mathbf{y}):=\|\mathbf{x}-\mathbf{y}\|_{1},
$$

the set of all points minimizing the expression $l(\mathbf{x}, n \cdot r)$ can be a closed interval, and taking as $s$ the middle point of this interval (in the case when a single real number is the minimizer, it can also be understood as a closed real interval), the median is obtained, $s=\operatorname{Med}(\mathbf{x})$. We modify this minimization problem replacing the metrics (distance functions) by dissimilarity functions and thus propose a new method for constructing aggregation functions.

Definition 2 Let $K: \mathbb{R} \rightarrow \mathbb{R}$ be a convex function with unique minimum $K(0)=0$. Then $K$ is called a shape function and for any real interval $\mathbb{I}$, the function $D_{K}: \mathbb{I}^{2} \rightarrow \mathbb{R}$ given by

$$
D_{K}(x, y):=K(x-y)
$$

is called a dissimilarity function.

Remark 2 (i) If the function $K$ in Definition 2 is even, then, taking $L$ : $\left[0, \infty\left[\rightarrow\left[0, \infty\left[\right.\right.\right.\right.$ the inverse function of $\left.K\right|_{[0, \infty}\left[\right.$, and defining $d_{K}: \mathbb{R}^{n} \rightarrow \mathbb{R}$ by

$$
d_{K}(\mathbf{x}, \mathbf{y}):=L\left(\sum_{i=1}^{n} K\left(x_{i}-y_{i}\right)\right),
$$

one can show that $d_{K}$ is a metric on $\mathbb{R}^{n}$. The above mentioned Euclidean metric $d$ is related to $K$ given by $K(x)=x^{2}$, while the "Manhattan metric" $l$ is related to $K(x)=|x|$. Similarly, the $L_{p}$-distance for $p \in[1, \infty[$ are related to $K_{(p)}(x):=|x|^{p}$.

(ii) Dissimilarity functions are a special kind of penalty functions introduced, discussed and applied in [10, 13, 48, 50, 73, 76].

Example 4 (i) For the shape function $K(x)=\exp (|x|)-1$, the corresponding dissimilarity function $D_{K}$ is given by $D_{K}(x, y)=\exp (|x-y|)-1$.

(ii) Shape functions need not be even. For any $\gamma \in] 0, \infty[$ different from 1 , the function $K_{<\gamma>}: \mathbb{R} \rightarrow \mathbb{R}$ given by

$$
K_{<\gamma>}(x):= \begin{cases}x & \text { if } x \geqslant 0 \\ -\gamma x & \text { otherwise }\end{cases}
$$

is a shape function which is not even.

Proposition 6 For a given $n \in \mathbb{N}$, let $K_{1}, \ldots, K_{n}: \mathbb{R} \rightarrow \mathbb{R}$ be given shape functions. For an arbitrary real interval $\mathbb{I}$ define a function $\mathrm{A}_{K_{1}, \ldots, K_{n}}: \mathbb{I}^{n} \rightarrow \mathbb{I}$ by

$$
\mathrm{A}_{K_{1}, \ldots, K_{n}}(\mathbf{x}):=\frac{s_{*}+s^{*}}{2},
$$

where

$$
\left[s_{*}, s^{*}\right]=\left\{u \in \mathbb{I} \mid \sum_{i=1}^{n} D_{K_{i}}\left(x_{i}, u\right)=\inf \left\{\sum_{i=1}^{n} D_{K_{i}}\left(x_{i}, r\right), r \in \mathbb{I}\right\}\right\} .
$$

Then $\mathrm{A}_{K_{1}, \ldots, K_{n}}$ is an idempotent aggregation function. 
Corollary 1 For any fixed shape function $K: \mathbb{R} \rightarrow \mathbb{R}$ and any real interval $\mathbb{I}$, the extended function $\mathrm{A}_{K}: \cup_{n \in \mathbb{N}} \mathbb{I}^{n} \rightarrow \mathbb{I}$ given by

$$
\mathrm{A}_{K}(\mathbf{x}):=\mathrm{A}_{K, \ldots, K}(\mathbf{x}),
$$

see (11), is a symmetric idempotent extended aggregation function.

Example 5 (i) For $n=2$, and for $x \in \mathbb{R}$, take $K_{1}(x)=|x|$ and $K_{2}(x)=x^{2}$, i.e., $K_{1}=K_{(1)}$ and $K_{2}=K_{(2)}$ are shape functions. Then $\mathrm{A}_{K_{1}, K_{2}}: \mathbb{R}^{2} \rightarrow$ $\mathbb{R}$ is given by

$$
\mathrm{A}_{K_{1}, K_{2}}(x, y)=\operatorname{Med}\left(x, y-\frac{1}{2}, y+\frac{1}{2}\right) .
$$

(ii) For $K_{<\gamma>}$ given in Example 4 (ii), the extended aggregation function $\mathrm{A}_{<\gamma>}: \cup_{n \in \mathbb{N}} \mathbb{R}^{n} \rightarrow \mathbb{R}$ is the $\frac{1}{1+\gamma} 100 \%$-quantile from the uniformly distributed sample $\left(x_{1}, \ldots, x_{n}\right)$, well known in statistics.

For each shape function $K, w K$ is also a shape function for any positive weight $w$. This fact allows one to easily incorporate weights into the $\mathrm{A}_{K_{1}, \ldots, K_{n}}$ introduced in Proposition 6, simply dealing with the aggregation function $\mathrm{A}_{w_{1} K_{1}, \ldots, w_{n} K_{n}}$. For any $w>0$,

$$
\mathrm{A}_{K_{1}, \ldots, K_{n}}=\mathrm{A}_{w K_{1}, \ldots, w K_{n}},
$$

and thus it suffices to deal with normalized weight vectors $\mathbf{w} \in[0,1]^{n}, \sum_{i=1}^{n} w_{i}=$ 1 , only.

Example 6 (i) For $\mathbf{w} \in[0,1]^{n}$ and $K=K_{(2)}$, we have $\mathrm{A}_{K_{(2)}}=\mathrm{AM}$ and

$$
\left(\mathrm{A}_{K}\right)_{\mathbf{w}}=\mathrm{A}_{w_{1 K}(2)}, \ldots, w_{n K}(2)
$$

is the weighted arithmetic mean with weights $w_{1}, \ldots, w_{n}$.

(ii) Using our approach, we can introduce weights in nonsymmetric aggregation, too. Continuing Example 5 (i), we have

$$
\begin{gathered}
\left(\mathbf{A}_{w_{1} K_{(1)}, w_{2} K_{(2)}}\right)(x, y)=\operatorname{Med}\left(x, y-\frac{w_{1}}{2 w_{2}}, y+\frac{w_{1}}{2 w_{2}}\right) \\
\left(\text { if } w_{2}=0, \text { formally } \operatorname{Med}(x, y-\infty, y+\infty)=x\right) .
\end{gathered}
$$

Remark 3 The fixed weights $w_{i}$ in the above approach (independent of input values $x_{i}$ ) can be replaced by input-dependent weights $w\left(x_{i}\right)$ for some weighting function $w: \mathbb{I} \rightarrow[0, \infty[$, i.e., we have to minimize the expression

$$
\sum_{i=1}^{n} w\left(x_{i}\right) D_{K_{i}}\left(x_{i}, r\right)
$$

Based on minimization of (13), we can introduce the (extended) function $\mathrm{A}_{K_{1}, \ldots, K_{n} ; w}$ : $\mathbb{I}^{n} \rightarrow \mathbb{I}$ (respectively, $\mathrm{A}_{K ; w}: \cup_{n \in \mathbb{N}} \mathbb{I}^{n} \rightarrow \mathbb{I}$ ) in the same way as we have introduced the function $\mathrm{A}_{K_{1}, \ldots, K_{n}}$ in (11). However, the monotonicity (and even idempotency) of these functions may fail, in general. Some more details on this topic can be found in $[49,50]$. 
Example 7 For $K=K_{(2)}$ and $\left.w: \mathbb{I} \rightarrow\right] 0, \infty\left[\right.$, the extended function $\mathrm{A}_{K ; w}$ : $\cup_{n \in \mathbb{N}} \mathbb{I}^{n} \rightarrow \mathbb{I}$ is given by

$$
\mathrm{A}_{K ; w}(\mathbf{x})=\frac{\sum_{i=1}^{n} w\left(x_{i}\right) x_{i}}{\sum_{i=1}^{n} w\left(x_{i}\right)}
$$

and it is called a mixture operator in [42]; see also [5]. On $\mathbb{I}=[0,1]$, if a smooth nondecreasing function $w$ satisfies $w(x) \geqslant w^{\prime}(x)$, then $\mathrm{A}_{K ; w}$ is an idempotent symmetric extended aggregation function. The above condition can be relaxed to $w(x) \geqslant w^{\prime}(x)(1-x)$ for all $x \in[0,1]$; see [49].

Remark 4 We can modify a dissimilarity function $D_{K}$ on an interval II by a continuous strictly monotone function $f: \mathbb{I} \rightarrow \mathbb{R}$ (this function is often called a scale) to

$$
D_{K, f}(x, y):=K(f(x)-f(y)) .
$$

However, the minimization problem $\sum_{i=1}^{n} D_{K_{i}, f}\left(x_{i}, r\right)$ leads then to an aggregation function $\mathrm{A}_{K_{1}, \ldots, K_{n} ; f}: \mathbb{I}^{n} \rightarrow \mathbb{I}$ (or an extended aggregation function $\mathrm{A}_{K ; f}:$ $\cup_{n \in \mathbb{N}} \mathbb{I}^{n} \rightarrow \mathbb{I}$ ) which is an $f$-transform of the aggregation function $\mathrm{A}_{K_{1}, \ldots, K_{n}}$ (extended aggregation function $\mathrm{A}_{K}$ ), i.e., we can look on this approach as a combination of two construction methods. As a typical example recall the extended aggregation function $\mathrm{A}_{K_{(2)} ; f}$ based on the shape function $K_{(2)}(x)=x^{2}$ which is, in fact, the quasi-arithmetic mean based on the generator $f$.

\subsection{Some other aggregation-based construction methods}

There are several construction methods which we have not discussed yet, which we recall only briefly. Among construction methods based on a given aggregation function possessing some additional property and yielding another aggregation function with desired property let us mention the idempotization and symmetrization. Important issue is the method of ordinal sums originally introduced by Birkhoff [6] in the framework of posets and lattices. There are well-known ordinal sums of t-norms, t-conorms (see [35]) and copulas (see [55]). As an interesting example of an ordinal sum of aggregation functions proposed by De Baets and Mesiar we give the next proposition.

Proposition 7 Let $0=a_{0}<a_{1}<\cdots<a_{n}=1$, and let $\mathrm{A}_{i}:\left[a_{i-1}, a_{i}\right]^{m} \rightarrow$ $\left[a_{i-1}, a_{i}\right], i=1, \ldots, n$, be continuous aggregation functions. Let $f:[0,1] \rightarrow \overline{\mathbb{R}}$ be a continuous strictly monotone non-bijective function. Then the mapping $\mathrm{A}:[0,1]^{m} \rightarrow[0,1]$ given by

$$
\mathrm{A}\left(x_{1}, \ldots, x_{m}\right)=f^{-1}\left(\sum_{i=1}^{n} f\left(\mathrm{~A}_{i}\left(x_{1}^{(i)}, \ldots, x_{m}^{(i)}\right)\right)-\sum_{i=1}^{n-1} f\left(a_{i}\right)\right),
$$

where $x^{(i)}=\operatorname{Max}\left(a_{i-1}, \operatorname{Min}\left(a_{i}, x\right)\right)$, is a continuous aggregation function.

Evidently, $\left.\mathrm{A}\right|_{\left[a_{i-1}, a_{i}\right]^{m}}=\mathrm{A}_{i}$, i.e., $\mathrm{A}$ extends all $\mathrm{A}_{i}$.

Further, $A$ is a t-norm, t-conorm, copula, quasi-copula whenever all $A_{i}$ are t-norms, t-conorms, copulas, quasi-copulas, respectively, i.e., Proposition 7 expresses in one formula, in general, different formulas for ordinal sums of $t$-norms, t-conorms, copulas and quasi-copulas. 
For special classes of aggregation functions, several extension methods are known based on a-priori given partial information. This is, e.g., the case of several integrals (Choquet, Sugeno, etc.), extending the knowledge of A for boolean inputs (i.e., a capacity) to the entire A, see also [29].

Another related approaches are known for t-norms (strict t-norm determined by diagonal section and opposite diagonal section, for example), for copulas (for known diagonal section), etc.

\section{Conjunctive aggregation functions}

As already mentioned in the introduction in [29], a conjunctive aggregation function is any aggregation function which is bounded from above by the Min, independently of the interval II we are dealing with. For the sake of transparency, and because of the fact that the majority of applications dealing with conjunctive aggregation functions is linked to the unit interval $\mathbb{I}=[0,1]$, we restrict our considerations in this section to the unit interval. Directly from the definition it follows that Min is the greatest conjunctive aggregation function (independently of arity, but also as an extended aggregation function). On the other hand, the smallest aggregation function $A_{\perp}$, which up to the boundary case $\mathrm{A}_{\perp}(1, \ldots, 1)=1$ coincide with the zero constant function, is also the smallest conjunctive aggregation function. In the next definition we recall several distinguished classes of binary conjunctive aggregation functions.

Definition 3 Let $\mathrm{A}:[0,1]^{2} \rightarrow[0,1]$ be an aggregation function.

(i) A is a boundary weak triangular norm (bwt-norm for short) if it is an associative symmetric conjunctive aggregation function.

(ii) If $\mathrm{A}$ has a neutral element $e=1$, then $\mathrm{A}$ is called a conjunctor.

(iii) A is a triangular norm (t-norm in short) if it is a bwt-norm and a conjunctor.

(iv) $\mathrm{A}$ is a quasi-copula if it is a conjunctive aggregation function and satisfies the Lipschitz condition with constant 1 , i.e., such that for all $x, y, u, v, \in$ $[0,1]$ we have

$$
|\mathrm{A}(x, y)-\mathrm{A}(u, v)| \leqslant|x-u|+|y-v| .
$$

(v) A is a copula if it is a conjunctor and fulfills the moderate growth property, i.e., such that for all $x, y, u, v \in[0,1]$, with $x \leqslant u, y \leqslant v$, we have

$$
\mathrm{A}(x, y)+\mathrm{A}(u, v) \geqslant \mathrm{A}(x, v)+\mathrm{A}(u, y) .
$$

Remark 5 We have the following comments related to Definition 3 with the same numberings as in Definition 3.

(i) Bwt-norms were considered in [56, 68].

(ii) Conjunctors are known under several different names, e.g., semicopulas [19], weak t-norms [64], etc. We call these aggregation functions conjunctors following [46]. Conjunctors are conjunctive aggregation functions. 
(iii) Triangular norms were introduced in [60], and for more details we recommend monographs [3, 35, 62]. These conjunctors model the intersection in the theory of fuzzy sets, and play a role of conjunctions in fuzzy logics.

(iv) Quasi-copulas were introduced [4] in relationship with copulas, and in its above form in [27]. They are applied in several domains, such as preference structures modeling or fuzzy logics.

(v) Copulas were introduced in [63], and more details can be found in monographs [33, 55, 62]. They form a proper subclass of quasi-copulas. Binary associative copulas and associative quasi-copulas coincide and they are just 1-Lipschitz triangular norms. They model the dependence structure of multivariate random variables.

Bwt-norms and t-norms are associative and thus their extensions to extended aggregation functions are trivial, hence we keep the same name and notation for the binary and the extended bwt-norms and t-norms. An extended conjunctor is an extended aggregation function on $[0,1]$ with extended neutral element $e=1$. Similarly, a 1-Lipschitzian conjunctive extended aggregation function is called a (extended) quasi-copula. A $n$-ary copula $C:[0,1]^{n} \rightarrow[0,1]$ (n-copula, in short) with $n>2$ is a quasi-copula satisfying the $n$-increasing monotonicity property, i.e., for any $\left(x_{1}, \ldots, x_{n}\right),\left(y_{1}, \ldots, y_{n}\right) \in[0,1]^{n}$ such that $x_{1} \leqslant y_{1}, \ldots, x_{n} \leqslant y_{n}$ it holds

$$
\sum_{\mathbf{d}}\left(\prod_{i=1}^{n} d_{i}\right) \mathrm{C}\left(u_{1}^{\left(d_{1}\right)}, \ldots, u_{n}^{\left(d_{n}\right)}\right) \geqslant 0,
$$

where the sum is taken over all $n$-tuples $\mathbf{d}=\left(d_{1}, \ldots, d_{n}\right) \in\{-1,1\}^{n}$ and where $u_{i}^{(-1)}:=x_{i}, u_{i}^{(1)}:=y_{i}$. A general copula $\mathrm{C}: \cup_{n=1}^{\infty}[0,1]^{n} \rightarrow[0,1]$ is an extended quasi-copula such that its $n$-ary version $\mathrm{C}^{(n)}:[0,1]^{n} \rightarrow[0,1]$ is an $n$-copula for each $n \geqslant 2$.

Examples of nondecreasing functions bounded by Min but not necessarily being conjunctive aggregation functions are triangular subnorms introduced by Jenei [32]; see [35, 51, 52].

Definition 4 A symmetric, nondecreasing, associative function $\mathrm{R}:[0,1]^{2} \rightarrow$ $[0,1]$ such that $\mathrm{R} \leqslant$ Min is called a triangular subnorm, t-subnorm in short.

The smallest t-subnorm is the constant 0 two-place function, while the greatest t-subnorm is Min. T-subnorms which are also aggregation functions are bwtnorms.

The relations between the introduced classes of conjunctive binary conjunctive aggregation are visualized in Figure 1.

In the class of conjunctive aggregation functions, several construction methods introduced in the previous section can be applied. We give some examples here.

Proposition 8 Let $\varphi$ be an increasing bijection from $[0,1]$ onto $[0,1]$ and let $\mathrm{A}$ be a conjunctive $n$-ary (extended) aggregation function. Then $\mathrm{A}_{\varphi}:[0,1]^{n} \rightarrow$ $[0,1]\left(\mathrm{A}_{\varphi}: \cup_{n \in \mathbb{N}}[0,1]^{n} \rightarrow[0,1]\right)$ given by

$$
\mathrm{A}_{\varphi}(\mathbf{x})=\varphi^{-1}\left(\mathrm{~A}\left(\varphi\left(x_{1}\right), \ldots, \varphi\left(x_{n}\right)\right)\right),
$$

(see Section 2.1) is a conjunctive extended aggregation function. 


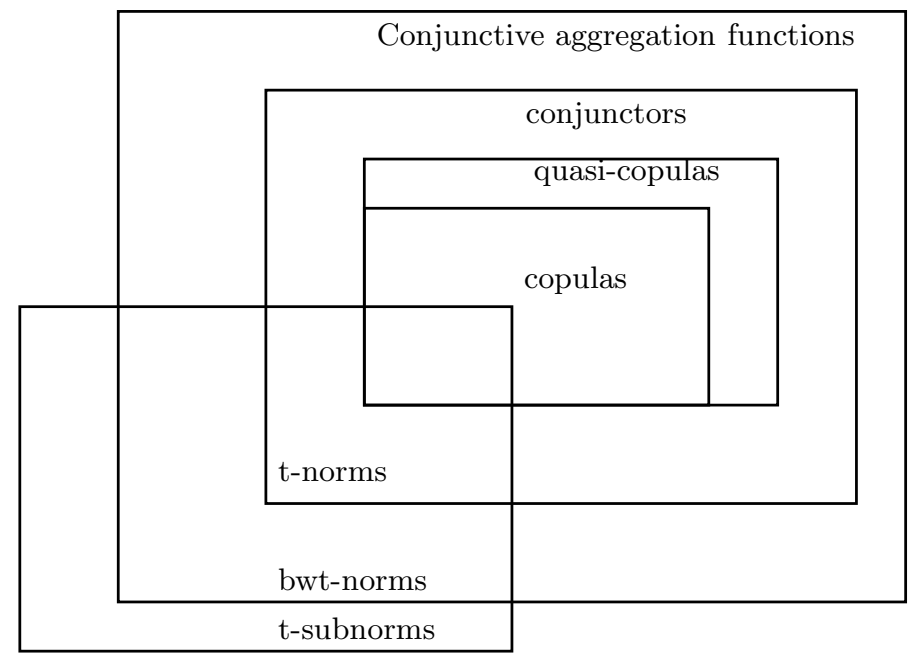

Figure 1: Relations between various particular binary conjunctive aggregation functions

Remark 6 (i) Proposition 8 remains true if we replace the conjunctive extended aggregation function by bwt-norms, $t$-norms or conjunctors. However, it fails for quasi-copulas and copulas, in general.

(ii) Any nondecreasing function $\mathrm{F}:[0,1]^{n} \rightarrow[0,1]$, bounded from above by Min, i.e., $\mathrm{F} \leqslant \mathrm{Min}$, can be redefined to a conjunctive aggregation function $\mathrm{F}^{\sim}$, simply by putting $\mathrm{F}^{\sim}(\mathbf{1})=1$ and $\mathrm{F}^{\sim}(\mathbf{x})=\mathrm{F}(\mathbf{x})$ in all other cases (so, for example, if $\mathrm{F}$ is identically zero, $\mathrm{F}^{\sim}$ is just the smallest aggregation function, $\mathrm{F}^{\sim}=\mathrm{A}_{\perp}$ ). This redefinition preserves the symmetry, but it may violate the associativity of binary or extended $\mathrm{F}$, in general.

Proposition 9 Let $\mathrm{A}$ be an idempotent m-ary (extended) aggregation function on $[0,1], \mathrm{A}_{1}, \ldots, \mathrm{A}_{m}$ be $n$-ary (extended) conjunctive aggregation functions on $[0,1]$. Then the composed $n$-ary aggregation function $\mathrm{B}:[0,1]^{n} \rightarrow[0,1]$ (extended aggregation function $\left.\mathrm{B}: \cup_{n \in \mathbb{N}}[0,1]^{n} \rightarrow[0,1]\right)$ given by

$$
B(x)=A\left(A_{1}(x), \ldots, A_{m}(x)\right)
$$

is an n-ary (extended) conjunctive aggregation function.

There are also other kinds of composition yielding conjunctive aggregation functions.

Proposition 10 Let $\mathrm{A}$ be a conjunctive m-ary (extended) aggregation function on $[0,1]$, and let $\mathrm{A}_{1}, \ldots, \mathrm{A}_{m}$ be $n$-ary (extended) aggregation functions on $[0,1]$ and there is at least one $i \in\{1, \ldots, m\}$ so that $\mathrm{A}_{i}$ is conjunctive. Then the composed $n$-ary aggregation function $\mathrm{B}:[0,1]^{n} \rightarrow[0,1]$ (extended aggregation function $\left.\mathrm{B}: \cup_{n \in \mathbb{N}}[0,1]^{n} \rightarrow[0,1]\right)$ given by

$$
\mathrm{B}(\mathrm{x})=\mathrm{A}\left(\mathrm{A}_{1}(\mathrm{x}), \ldots, \mathrm{A}_{m}(\mathbf{x})\right)
$$

is an n-ary conjunctive (extended) aggregation function. 
Proposition 11 Let $\mathrm{A}:[0,1]^{2} \rightarrow[0,1]$ be a conjunctive or an idempotent aggregation function, and let $\mathrm{B}:[0,1]^{m} \rightarrow[0,1]$ and $\mathrm{C}:[0,1]^{n} \rightarrow[0,1]$ be two conjunctive aggregation functions. Then the function $\mathrm{D}:[0,1]^{m+n} \rightarrow[0,1]$ given by

$$
\mathrm{D}\left(x_{1}, \ldots, x_{m+n}\right)=\mathrm{A}\left(\mathrm{B}\left(x_{1}, \ldots, x_{m}\right), \mathrm{C}\left(x_{m+1}, \ldots, x_{m+n}\right)\right)
$$

is an $(m+n)$-ary conjunctive aggregation function.

Another distinguished construction method preserving conjunctive aggregation functions is the conjunctive ordinal sum.

Proposition 12 Let (]$a_{k}, b_{k}[)_{k \in K}$ be a disjoint system of open subintervals of $[0,1]$, and let $\left(\mathrm{A}_{k}\right)_{k \in K}$ be a system of conjunctive n-ary (extended) aggregation functions on $[0,1]$ (of conjunctors, of triangular norms, of quasi-copulas, of copulas, respectively). Then the mapping $\mathrm{B}:[0,1]^{n} \rightarrow[0,1]\left(\mathrm{B}: \cup_{n \in \mathbb{N}}[0,1]^{n} \rightarrow\right.$ $[0,1])$ given by

$\mathrm{B}\left(x_{1}, \ldots, x_{n}\right)= \begin{cases}\mathrm{A}_{k}\left(\min \left(x_{1}, b_{k}\right), \ldots, \min \left(x_{n}, b_{k}\right)\right) & \left.\text { if } \operatorname{Min}\left(x_{1}, \ldots, x_{k}\right) \in\right] a_{k}, b_{k}[, \\ \operatorname{Min}\left(x_{1}, \ldots, x_{n}\right) & \text { else, }\end{cases}$

is an n-ary (extended) conjunctive aggregation function (a conjunctor, triangular norm, quasi-copula, copula, respectively).

The ordinal sum aggregation function $B$ introduced in the last Proposition 12 is often denoted as $\mathrm{B}=\left(<a_{k}, b_{k}, \mathrm{~A}_{k}>\mid k \in K\right)$. Formally, we allow also an empty index set $K$, in which case $\mathrm{B}=$ Min by definition.

Though we have introduced several classes of conjunctive aggregation functions, we will put a closer look only on the two most important classes, namely triangular norms and copulas. More details about the remaining classes of conjunctive aggregation functions can be found in Chapter 3 in [28].

\subsection{Triangular norms}

Definition 3 (iii) gives us the binary t-norm $\mathrm{T}^{(2)}:[0,1]^{2} \rightarrow[0,1]$. Associativity of t-norms $\mathrm{T}^{(2)}:[0,1]^{2} \rightarrow[0,1]$ allows us to consider their extensions to $n$-ary functions $\mathrm{T}^{(n)}:[0,1]^{n} \rightarrow[0,1]$ in the following way

$$
\mathbf{T}^{(n)}(\mathbf{x}):=\mathbf{T}^{(2)}\left(\mathbf{T}^{(n-1)}\left(x_{1}, \ldots, x_{n-1}\right), x_{n}\right) .
$$

So we can give the definition of a t-norm in extended form.

Definition 5 An extended t-norm $\mathrm{T}: \cup_{n \in \mathbb{N}}[0,1]^{n} \rightarrow[0,1]$ is an associative symmetric extended aggregation function with extended neutral element 1.

The smallest t-norm (and also the smallest conjunctor) is the drastic product $\mathbf{T}_{\mathbf{D}}$ (independently of the arity) given by

$$
\mathrm{T}_{\mathbf{D}}\left(x_{1}, \ldots, x_{n}\right)= \begin{cases}x_{i} & \text { if for each } j \neq i, x_{j}=1 \\ 0 & \text { else }\end{cases}
$$

Proposition 13 (i) Each t-norm $\mathrm{T}$ is an aggregation function with annihilator 0 , i.e., $\mathbf{T}(\mathbf{x})=0$ for all $\mathbf{x} \in[0,1]^{n}$ such that $0 \in\left\{x_{1}, \ldots, x_{n}\right\}$. 
(ii) The greatest (and the only idempotent) t-norm is the standard Min, i.e., for any t-norm $\mathrm{T}$,

$$
\mathrm{T}_{\mathrm{D}} \leqslant \mathrm{T} \leqslant \text { Min }
$$

$T_{\mathbf{D}}$ and $T_{\mathbf{M}}$ are basic t-norms. The next two basic t-norms are the standard product $T_{\mathbf{P}}=\Pi$, and the Eukasiewicz t-norm $T_{\mathbf{L}}$ given by

$$
\mathrm{T}_{\mathbf{L}}(\mathbf{x}):=\operatorname{Max}\left(0, \sum_{i=1}^{n} x_{i}-(n-1)\right) .
$$

Archimedean property plays a distinguished role in the domain of triangular norms. A t-norm $T$ is Archimedean whenever for each $x, y \in] 0,1[$ there is $n \in \mathbb{N}$ such that $\mathbf{T}^{(n)}(x, \ldots, x)<y$. For continuous t-norm $\mathbf{T}$, its Archimedeanity is equivalent to the diagonal inequality $\mathrm{T}(x, x)<x$ for all $x \in] 0,1$ [. Continuous Archimedean t-norms can be seen as an isomorphic transformation of the standard (truncated) addition.

Theorem 1 Let $\mathrm{T}: \cup_{n=1}^{\infty}[0,1]^{n} \rightarrow[0,1]$ be a triangular norm. Then $\mathrm{T}$ is Archimedean if and only if there is a continuous strictly decreasing function $h:[0,1] \rightarrow[0, \infty], h(1)=0$, so that

$$
\mathrm{T}\left(x_{1}, \ldots, x_{n}\right)=h^{-1}\left(\min \left(h(0), \sum_{i=1}^{n} h\left(x_{i}\right)\right)\right) .
$$

Note that $h$ generating $\mathrm{T}$ via (16) is called an additive generator of $\mathrm{T}$ and it is unique up to a multiplicative constant (hence, fixing $h(0.5)=1$, the relationship between continuous Archimedean t-norms and additive generators is one-toone). Moreover, defining a function $g:[0,1] \rightarrow[0,1]$ by $g(x)=\exp (-h(x))$, a multiplicative version of (16) is obtained,

$$
\mathrm{T}\left(x_{1}, \ldots, x_{n}\right)=g^{-1}\left(\max \left(g(0), \prod_{i=1}^{n} g\left(x_{i}\right)\right)\right) .
$$

The function $g$ generating $\mathrm{T}$ via (17) is called a multiplicative generator of $\mathrm{T}$, and it is unique up to a positive power. Continuous Archimedean t-norms can be divided into two classes:

(i) strict t-norms are continuous t-norms which are strictly increasing (as binary functions) on $] 0,1]^{2}$ and they are characterized by unbounded additive generators, i.e., $h(0)=\infty$. Note that they are just isomorphic transformations of the product $\mathrm{t}$-norm $\Pi$, i.e., $\mathrm{T}=\Pi_{\phi}$ for some increasing bijection $\phi:[0,1] \rightarrow[0,1]$. Observe that then $\phi=g$ is just the multiplicative generator of $\mathrm{T}$;

(ii) nilpotent t-norms are non-strict continuous Archimedean t-norms, they are characterized by bounded additive generators, and to ensure the uniqueness of these generators, often the normed additive generators satisfying $h(0)=1$ are considered; nilpotent t-norms are isomorphic to the Eukasiewicz t-norm $T_{\mathbf{L}}$, i.e., $\mathbf{T}=\left(\mathrm{T}_{\mathbf{L}}\right)_{\phi}$ for some increasing bijection $\phi:[0,1] \rightarrow[0,1]$. 
The importance of Archimedean t-norms in the framework of continuous triangular norms is illustrated in the next two theorems.

Theorem 2 Let $\mathrm{T}: \cup_{n=1}^{\infty}[0,1]^{n} \rightarrow[0,1]$ be a triangular norm. Then the following are equivalent:

(i) $\mathrm{T}$ is continuous;

(ii) $\mathrm{T}$ is isomorphic to an ordinal sum $\left(<a_{k}, b_{k}, \mathrm{~T}_{k}>\mid k \in K\right)$ where for each $k \in K, \mathbf{T}_{k} \in\left\{\Pi, \mathbf{T}_{\mathbf{L}}\right\}$

(iii) $\mathrm{T}$ is an ordinal sum $\left(<a_{k}, b_{k}, \mathrm{~T}_{k}>\mid k \in K\right)$ where for each $k \in K, \mathrm{~T}_{k}$ is a continuous Archimedean t-norm;

(iv) there is a system (]$a_{k}, b_{k}[)_{k \in K}$ of disjoint open subintervals of $] 0,1[$ and a system $\left(h_{k}\right)_{k \in K}$ of continuous strictly decreasing functions $h_{k}:\left[a_{k}, b_{k}\right] \rightarrow$ $[0, \infty]$ satisfying $h_{k}\left(b_{k}\right)=0$ so that

$$
\mathrm{T}\left(x_{1}, \ldots, x_{n}\right)=\left\{\begin{array}{l}
h_{k}^{-1}\left(\min \left(h_{k}(0), \sum_{i=1}^{n} h_{k}\left(\min \left(x_{i}, b_{k}\right)\right)\right)\right. \\
\text { if } \left.\operatorname{Min}\left(x_{1}, \ldots, x_{n}\right) \in\right] a_{k}, b_{k}[ \\
\operatorname{Min}\left(x_{1}, \ldots, x_{n}\right)
\end{array}\right.
$$

else.

Theorem 3 Let $\mathrm{T}$ be a continuous triangular norm. Then for any fixed $n \geqslant 2$ and any $\varepsilon>0$ there is a strict t-norm $\mathrm{T}_{1}$ and a nilpotent $t$-norm $\mathrm{T}_{2}$ such that for $i=1,2$ it holds

$$
\sup \left\{\left|\mathrm{T}(x, y)-\mathrm{T}_{i}(x, y)\right| \mid(x, y) \in[0,1]^{2}\right\} \leqslant \varepsilon .
$$

Due to Theorem 3, a general continuous t-norm can be approximated (with any given tolerance $\varepsilon$ ) by a strict t-norm or by a nilpotent t-norm.

The fact that the complete information about a continuous Archimedean t-norm $\mathrm{T}$ is contained in its additive generator $h$ allows to transform the investigation of these t-norms into the study of the corresponding additive generators. For example, for comparison of continuous Archimedean t-norms we have the next result.

Proposition 14 Let $\mathrm{T}_{1}, \mathrm{~T}_{2}$ be two continuous Archimedean t-norms with the corresponding additive generators $h_{1}, h_{2}$. Then $\mathrm{T}_{1} \leqslant \mathrm{~T}_{2}$ if and only if the composite function $h_{1} \circ h_{2}^{-1}:\left[0, h_{2}(0)\right] \rightarrow[0, \infty]$ is subadditive, i.e., if for all $x, y \in\left[0, h_{2}(0)\right]$ such that also $x+y \leqslant h_{2}(0)$ it holds

$$
h_{1} \circ h_{2}^{-1}(x+y) \leqslant h_{1} \circ h_{2}^{-1}(x)+h_{1} \circ h_{2}^{-1}(y) .
$$

Note that the concavity of $h_{1} \circ h_{2}^{-1}$ is sufficient to ensure $T_{1} \leqslant T_{2}$.

For any fixed continuous Archimedean (strict, nilpotent) t-norm $T$ with an additive generator $h$, also $\left.h^{\lambda}, \lambda \in\right] 0, \infty[$ is an additive generator of a continuous Archimedean (strict, nilpotent) t-norm $T^{(\lambda)}$. Observe that the limit members of families $\left(T^{(\lambda)}\right)$ are $T^{(\infty)}=T_{M}$ and $T^{(0)}=T_{D}$, independently of the original fixed t-norm $\mathbf{T}$. For example, consider the nilpotent basic t-norm $\mathbf{T}_{\mathbf{L}}$ generated by a normed additive generator $h_{\mathbf{L}}:[0,1] \rightarrow[0,1], h_{\mathbf{L}}(x)=1-x$. Then

$$
\mathrm{T}_{\mathbf{L}}^{(\lambda)}\left(x_{1}, \ldots, x_{n}\right)=\max \left(0,1-\left(\sum_{i=1}^{n}\left(1-x_{i}\right)^{\lambda}\right)^{1 / \lambda}\right) .
$$


Note that these nilpotent t-norms are known as Yager t-norms [72], $\mathrm{T}_{\mathbf{L}}^{(\lambda)}=\mathrm{T}_{\lambda}^{Y}$. Similarly, consider the strict basic t-norm $\Pi$ with $h_{\Pi}(x)=-\log x$. Then the corresponding strict t-norms $\left.(\Pi)^{(\lambda)}=\mathrm{T}_{\lambda}^{A A}, \lambda \in\right] 0, \infty[$, are known as AczélAlsina t-norms [2] and they are given by

$$
\mathrm{T}_{\lambda}^{A A}\left(x_{1}, \ldots, x_{n}\right)=\exp \left(-\left(\sum_{i=1}^{n}\left(-\log x_{i}\right)^{\lambda}\right)^{1 / \lambda}\right) .
$$

Another modification of a fixed additive generator yielding a new additive generator is linked to the power of the argument. Indeed, for a fixed additive generator $h:[0,1] \rightarrow[0, \infty]$ of a given continuous Archimedean t-norm T, also the function $h_{(\lambda)}:[0,1] \rightarrow[0, \infty]$ given by $h_{(\lambda)}(x)=h\left(x^{\lambda}\right)$ is an additive generator of a t-norm denoted as $\mathrm{T}_{(\lambda)}$ whenever $\left.\lambda \in\right] 0, \infty[$. As a prototypical example for this construction of continuous Archimedean t-norms recall the Schweizer-Sklar t-norms $\left(\mathrm{T}_{\lambda}^{S S}\right)_{\lambda \in[-\infty, \infty]}$ introduced in [61] and which contains all four basic tnorms $\mathrm{T}_{\mathbf{M}}=\mathrm{T}_{-\infty}^{S S}, \Pi=\mathrm{T}_{0}^{S S}, \mathrm{~T}_{\mathbf{L}}=\mathrm{T}_{1}^{S S}$ and $\mathrm{T}=\mathrm{T}_{\infty}^{S S}$. For $\left.\lambda \in\right]-\infty, 0[\cup] 0, \infty[$, the continuous Archimedean t-norms $T_{\lambda}^{S S}$ are generated by additive generators $h_{\lambda}^{S S}:[0,1] \rightarrow[0, \infty]$ given by

$$
h_{\lambda}^{S S}(x)=\frac{1-x^{\lambda}}{\lambda} .
$$

Then evidently all nilpotent members of this family are related to the Eukasiewicz t-norm $\mathrm{T}_{L}, \mathrm{~T}_{\lambda}^{S S}=\left(\mathrm{T}_{\mathbf{L}}\right)_{(\lambda)}$ whenever $\left.\lambda \in\right] 0, \infty[$. Moreover, the strict members of this family are related to the Hamacher product $\mathrm{T}^{H}$ introduced in [31] and given (in binary form) by

$$
\mathrm{\top}^{H}(x, y)= \begin{cases}\frac{x y}{x+y-x y} & \text { if } x>0 \\ 0 & \text { else. }\end{cases}
$$

Note that the Hamacher product $\mathrm{T}^{H}$ is generated by an additive generator $h_{H}:[0,1] \rightarrow[0, \infty]$ given by $h_{H}(x)=x^{-1}-1$, and that $\mathrm{T}_{\lambda}^{S S}=\left(\mathrm{T}^{H}\right)_{-\lambda}$ whenever $\lambda \in]-\infty, 0[$.

Among several construction of non-continuous t-norms recall the next one.

Proposition 15 Let $E$ be subset of $[0,1]^{2}$ such that

(i) if $(x, y) \in E$ then also $(y, x) \in E$;

(ii) if $(x, y) \in E$ and $0 \leqslant u \leqslant x, 0 \leqslant v \leqslant y$, then also $(u, v) \in E$;

(iii) if $(x, y) \in[0,1]^{2}$ and $1 \in\{x, y\}$ then $(x, y) \in E$ if and only if $0 \in\{x, y\}$.

Then the function $\mathrm{T}_{E}:[0, a]^{2} \rightarrow[0,1]$ given by

$$
\mathrm{\top}_{E}(x, y)= \begin{cases}0 & \text { if }(x, y) \in E \\ \min (x, y) & \text { else }\end{cases}
$$

is a (binary) triangular norm.

Evidently, the only continuous t-norm $\mathrm{T}_{E}$ is the basic t-norm $\mathrm{T}_{\mathbf{M}}$ corresponding to the minimal $\left.\left.E_{*}=[0,1]^{2}-\right] 0,1\right]^{2}$. The smallest t-norm $\mathrm{T}_{E}$ is the 
basic t-norm $\mathbf{T}_{\mathbf{D}}$ corresponding to the maximal $E^{*}=\left[0,1\left[{ }^{2} \cup\{(0,1),(1,0)\}\left(\mathbf{T}_{D}\right.\right.\right.$ is right-continuous). A distinguished example of a left-continuous t-norm $\mathrm{T}_{E}$ is the nilpotent minimum $\mathbf{T}^{n M}$ introduced in [20] related to $E=\left\{(x, y) \in[0,1]^{2} \mid\right.$ $x+y \leqslant 1\}$,

$$
\mathrm{T}^{n M}(x, y)= \begin{cases}0 & \text { if } x+y \leqslant 1, \\ \min (x, y) & \text { else. }\end{cases}
$$

There are several peculiar triangular norm. For example, Krause t-norm discussed in details in [35][Appendix B1] is not continuous though its diagonal section is continuous (for any arity). The next t-norm $T:[0,1]^{2} \rightarrow[0,1]$ given by

$$
\mathbf{T}(x, y)= \begin{cases}\frac{x+y-1}{1-4(1-x)(1-y)} & \text { if } x+y>1 \\ 0 & \text { else }\end{cases}
$$

has unique discontinuity point $(0.5,0.5)$ (and it is left-continuous, see Example 3.72(i) in [35]). Many other examples, as well as a deep discussion of many aspects of the theory and application of triangular norms can be found in monographs $[3,35]$, but also in [28].

\subsection{Copulas}

Copulas were introduced by A.Sklar [63] in the multivariate statistical framework as "products" for distribution functions. $n$-ary copulas are characterized by the nonnegative probability of the $n$-dimensional box $\left[x_{1}, y_{1}\right] \times\left[x_{2}, y_{2}\right] \times \cdots \times$ $\left[x_{n}, y_{n}\right]$, which we shall denote by $[\mathbf{x}, \mathbf{y}]$. For $\mathbf{x}, \mathbf{y} \in[0,1]^{n}$ such that $\mathbf{x} \leqslant \mathbf{y}$, let $[\mathbf{x}, \mathbf{y}]$ be the corresponding $n$-box, and let $\mathbf{z}=\left(z_{1}, \ldots, z_{n}\right)$ be a vertex of $[\mathbf{x}, \mathbf{y}]$. Then we define $\operatorname{sign}_{[\mathbf{x}, \mathbf{y}]}(\mathbf{z})$ in the following way

$$
\operatorname{sign}_{[\mathbf{x}, \mathbf{y}]}(\mathbf{z}):= \begin{cases}1 & \text { if } z_{m}=x_{m} \text { for an even number of } m \text { 's } \\ -1 & \text { if } z_{m}=x_{m} \text { for an odd number of } m \text { 's. }\end{cases}
$$

If the vertices of the $n$-box $[\mathbf{x}, \mathbf{y}]$ are not all distinct, then $\operatorname{sign}_{[\mathbf{x}, \mathbf{y}]}(\mathbf{z})=0$.

Definition 6 For a fixed $n \geqslant 2$, let $\mathrm{C}:[0,1]^{n} \rightarrow[0,1]$ be an n-ary aggregation function with a neutral element $e=1$, such that for all $\mathbf{x}, \mathbf{y} \in[0,1]^{n}, \mathbf{x} \leqslant \mathbf{y}$, the following inequality (called $n$-increasing monotonicity or moderate growth) is fulfilled:

$$
\sum \operatorname{sign}_{[\mathbf{x}, \mathbf{y}]}(\mathbf{z}) \mathrm{C}(\mathbf{z}) \geqslant 0
$$

where the sum is taken over all vertices $\mathbf{z}$ of $[\mathbf{x}, \mathbf{y}]$. Then $\mathbf{C}$ is called an $n$-copula.

An extended aggregation function $\mathrm{C}: \cup_{n \in \mathbb{N}}[0,1]^{n} \rightarrow[0,1]$ such that for each $n \geqslant 2$ the corresponding $n$-ary aggregation function $\mathrm{C}^{(n)}$ is an $n$-copula is called a general copula.

To better understand the statistical background of copulas we recall here the Sklar theorem introduced in [63].

Theorem 4 (i) If $\mathrm{H}:[-\infty, \infty]^{n} \rightarrow[0,1]$ is an $n$-dimensional (cumulative) distribution function with one-dimensional marginal (cumulative) distribution functions $\mathrm{F}_{1}, \ldots, \mathrm{F}_{n}:[-\infty, \infty] \rightarrow[0,1]$, then there exists an $n$-copula C such that

$$
\mathrm{H}(\mathbf{x})=\mathrm{C}\left(\mathrm{F}_{1}\left(x_{1}\right), \ldots, \mathrm{F}_{n}\left(x_{n}\right)\right)
$$


for all $\mathbf{x} \in \mathbb{R}^{n}$. If $\mathrm{F}_{1}, \ldots, \mathrm{F}_{n}$ are continuous, then $\mathrm{C}$ is unique; otherwise $\mathrm{C}$ is uniquely determined on $\operatorname{ran}\left(\mathrm{F}_{1}\right) \times \cdots \times \operatorname{ran}\left(\mathrm{F}_{n}\right)$.

(ii) For any one-dimensional distribution functions $\mathrm{F}_{1}, \ldots, \mathrm{F}_{n}$, and any $n$ copula $\mathrm{C}$, the function $\mathrm{H}$ given by (19) is an $n$-dimensional distribution function with one-dimensional marginals $\mathrm{F}_{1}, \ldots, \mathrm{F}_{n}$.

Remark 7 (i) Each $n$-copula can be seen as (the restriction to $[0,1]^{n}$ of) an $n$-dimensional distribution function with marginals which have uniform distribution on $[0,1]$.

(ii) A nice probabilistic characterization of the three basic continuous $t$-norms $\left(\mathrm{T}_{\mathbf{L}}, \Pi\right.$, and $\left.\mathrm{Min}\right)$, which are also copulas, is the next one: For events $E_{1}, \ldots, E_{n}$ of the form $E_{i}:=\left\{X_{i} \leqslant x\right\}$, let $P\left(E_{1}\right), \ldots, P\left(E_{n}\right)$ be their respective probabilities. What can we say about the probability of the intersection $\bigcap_{i=1}^{n} E_{i}$ ?

The probability $P\left(E_{1} \cap \cdots \cap E_{n}\right)$ can be computed using Theorem 4 by means of a (in most cases unknown) copula $\mathrm{C}$,

$$
P\left(E_{1} \cap \cdots \cap E_{n}\right)=\mathrm{C}\left(P\left(E_{1}\right), \ldots, P\left(E_{n}\right)\right) .
$$

Due to the fact that $\mathrm{T}_{\mathbf{L}}<\mathrm{C} \leqslant$ Min for any copula $\mathrm{C}$ we have the (best) estimation

$$
\mathrm{T}_{\mathbf{L}}\left(P\left(E_{1}\right), \ldots, P\left(E_{n}\right)\right)<P\left(E_{1} \cap \cdots \cap E_{n}\right) \leqslant \operatorname{Min}\left(P\left(E_{1}\right), \ldots, P\left(E_{n}\right)\right) .
$$

If the events $E_{1}, \ldots, E_{n}$ are jointly independent, then $\mathrm{C}=\Pi$ and

$$
P\left(E_{1} \cap \cdots \cap E_{n}\right)=\Pi\left(P\left(E_{1}\right), \ldots, P\left(E_{n}\right)\right) .
$$

(iii) In the case described in Theorem 4 (ii), the left-hand side of the inequality (18) is exactly the probability of the n-dimensional box $[\mathbf{x}, \mathbf{y}]$ (which is clearly nonnegative).

We have the following basic properties of $n$-copulas, [62].

Proposition 16 Let $\mathrm{C}:[0,1]^{n} \rightarrow[0,1]$ be an $n$-copula. Then the following holds.

(i) Let $i \in[n]$. Then for all $x, y \in[0,1]$ and all $\mathbf{x} \in[0,1]^{n}$,

$$
\left|\mathrm{C}\left(x_{1}, \ldots, x_{i-1}, x, x_{i+1}, \ldots, x_{n}\right)-\mathrm{C}\left(x_{1}, \ldots, x_{i-1}, y, x_{i+1}, \ldots, x_{n}\right)\right| \leqslant|x-y| .
$$

(ii) For any $\mathbf{x}, \mathbf{y} \in[0,1]^{n}$ we have

$$
|\mathrm{C}(\mathbf{x})-\mathrm{C}(\mathbf{y})| \leqslant \sum_{i=1}^{n}\left|x_{i}-y_{i}\right|
$$

i.e., C is 1-Lipchitzian and therefore a continuous aggregation function. 
We introduce some examples of copulas based on statistical properties of random variables.

(i) As we have already noticed, the independence of random variables is expressed by the product copula $\Pi$, i.e., any $n$-tuple $\left(X_{1}, \ldots, X_{n}\right)$ of $n$ independent random variables has joint distribution

$$
F_{X_{1}, \ldots, X_{n}}(\mathbf{x})=\prod_{i=1}^{n} F_{X_{i}}\left(x_{i}\right) .
$$

(ii) As already mentioned above, another general copula is Min. Min relates random variables which are totally positively dependent in the sense that in the random vector $\left(X_{1}, \ldots, X_{n}\right)$, each $X_{i}$ can be expressed in the form $X_{i}=f_{i}\left(X_{1}\right)$, where $f_{i}$ is a real function increasing on the range of $X_{1}$. Then

$$
F_{X_{1}, \ldots, X_{n}}(\mathbf{x})=\operatorname{Min}\left(F_{X_{1}}\left(x_{1}\right), \ldots, F_{X_{n}}\left(x_{n}\right)\right) .
$$

(iii) In the case of 2-copulas, the smallest 2-copula $T_{\mathbf{L}}$ relates two negatively dependent random variables $X_{1}$ and $X_{2}, X_{2}=f\left(X_{1}\right)$, where $f$ is a real function decreasing on the range of $X_{1}$.

Due to Moynihan [54] it is known that associative binary copulas are exactly 1-Lipschitz triangular norms, i.e., they can be seen as ordinal sums of Archimedean copulas. Moreover, the next result was shown in [54].

Theorem 5 An aggregation function $\mathrm{C}:[0,1]^{2} \rightarrow[0,1]$ is an Archimedean copula if and only if it is a continuous triangular norm with a convex additive generator $h:[0,1] \rightarrow[0, \infty]$.

Due to the associativity, each 1-Lipschitz t-norm can be extended to $n$-ary (extended) 1-Lipschitz triangular norm, i.e., to $n$-ary (extended) quasi-copula. However, not all such aggregation functions are $n$-ary copulas. For example, taking the smallest binary copula $\mathbf{T}_{\mathbf{L}}$, its ternary form $\mathbf{T}_{\mathbf{L}}:[0,1]^{3} \rightarrow[0,1]$ is given by $\mathrm{T}_{\mathbf{L}}(x, y, z)=\max (0, x+y+z-2)$. For $\mathbf{x}=(0.5,0.5,0.5)$ and $\mathbf{y}=(1,1,1)$, the 3 -increasing property of $\mathbf{T}_{\mathbf{L}}$ would mean

$$
\begin{gathered}
\mathrm{T}_{\mathbf{L}}(1,1,1)-\left(\mathrm{T}_{\mathbf{L}}(0.5,1,1)+\mathrm{T}_{\mathbf{L}}(1,0.5,1)+\mathrm{T}_{\mathbf{L}}(1,1,0.5)\right)+\mathrm{T}_{\mathbf{L}}(0.5,0.5,1) \\
+\mathrm{T}_{\mathbf{L}}(0.5,1,0.5)+\mathrm{T}_{\mathbf{L}}(1,0.5,0.5)-\mathrm{T}_{\mathbf{L}}(0.5,0.5,0.5) \geqslant 0
\end{gathered}
$$

what obviously is not true. Therefore, $\mathrm{T}_{\mathbf{L}}$ is not a ternary copula! Though there is no smallest $n$-copula for $n>2$, due to [44] we have the next result.

Theorem 6 Let $n>2$. An $n$-ary extension $\mathrm{C}:[0,1]^{n} \rightarrow[0,1]$ of a continuous Archimedean t-norm $\mathrm{T}$ with an additive generator $h$ is an $n$-copula if and only if the function $q:[-\infty, 0] \rightarrow[0,1]$ given by

$$
q(x)=h^{-1}(\min (h(0),-x))
$$

has on $]-\infty, 0[$ all derivatives of order $1, \ldots, n-2$ which are non-negative and the $(n-2)$ th derivative of $q$ is a convex function. The smallest $n$-ary copula $\mathrm{C}_{n}$ of this type has an additive generator $h_{n}:[0,1] \rightarrow[0, \infty]$ given by $h_{n}(x)=1-x^{1 /(n-1)}$, i.e., $\mathrm{C}_{n}=\mathrm{T}_{1 /(n-1)}^{S S}$. 
It is not difficult to see that the $n$-ary copula $\mathrm{C}_{n}$ is the smallest one in the class of all $n$-ary copulas which are $n$-ary extensions of an associative copula. Based on Theorem 6 , it is also visible that the smallest associative general copula is the extended product $\Pi$ (recall that $\Pi=\mathrm{T}_{0}^{S S}$ ).

One crucial difference between the classes of (binary, $n$-ary, extended) tnorms and copulas is the convexity and compactness of copula classes [36]. For example, the function $D=\frac{T_{M}+\Pi}{2}$ is a copula for any arity (and thus also a general copula, though it is not associative).

From the introduced parametric families of continuous Archimedean t-norms, observe that the Aczel-Alsina t-norms $\mathrm{T}_{\lambda}^{A A}$ are binary copulas (even general copulas) for any $\lambda \geqslant 1$ (and in the copula theory they are known as Gumbel family). Similarly, Yager t-norms $\mathrm{T}_{\lambda}^{Y}$ are binary copulas for any $\lambda \geqslant 1$. Moreover, $\mathrm{T}_{\lambda}^{Y}$ as $n$-ary aggregation function is an $n$-copula if and only if $\lambda \geqslant n-1$. In the case of Schweizer-Sklar t-norms, these are (as $n$-ary functions) $n$-copulas whenever $\lambda \leqslant \frac{1}{n-1}$, and they (as extended aggregation functions) are general copulas for each $\lambda \leqslant 0$. In the copula world, copula members of Schweizer-Sklar family they are known as Clayton copulas.

In the following proposition we introduce an important subclass of 2-copulas, namely the Archimax copulas [16].

Proposition 17 Let $t:[0,1] \rightarrow[0, \infty]$ be a convex decreasing function such that $t(1)=0$ (i.e., $t$ is an additive generator of some 2-copula $C_{t}$ ), and let $D:[0,1] \rightarrow[0,1]$ be a convex function bounded from below by $\operatorname{Max}(x, 1-x)$. (D is a so-called dependence function.) Then the mapping $\mathrm{C}_{t, D}:[0,1]^{2} \rightarrow[0,1]$ given by $($ for $(x, y) \in] 0,1\left[^{2}\right)$

$$
\mathrm{C}_{t, D}(x, y)=t^{-1}\left(\operatorname{Min}\left(t(0),(t(x)+t(y)) D\left(\frac{t(x)}{t(x)+t(y)}\right)\right)\right)
$$

is a 2-copula. This copula is called an Archimax copula.

The smallest Archimax copula with fixed $t$ corresponds to the constant dependence function $D=1$, and then $C_{t, 1}=C_{t}$ is an Archimedean copula generated by $t$. On the other hand, for the lowest dependence function $D_{*}(x):=\operatorname{Max}(x, 1-x)$, we have $C_{t, D_{*}}=\operatorname{Min}^{(2)}$, independently of $t$. More details about Archimax copulas can be found also in [37,38]. In the special case $t(x)=-\log x$ (i.e., when $C_{t}=\Pi$ ), the corresponding Archimax copulas $\mathrm{C}_{t, D}$ are known in statistics as Max-attractor copulas $[15,26,66]$ and they are characterized by power stability, i.e.,

$$
\mathrm{C}_{t, D}\left(x^{p}, y^{p}\right)=\left(\mathrm{C}_{t, D}(x, y)\right)^{p}
$$

for all $p \in] 0, \infty[$.

Max-attractors are closed under Max-composition and under weighted geometric mean composition.

For a deeper discussion on copulas, their statistical and functional properties we recommend specialized monographs $[33,55]$ and $[3,35,62]$.

\section{Disjunctive aggregation functions}

As already mentioned, disjunctive aggregation functions are those which are bounded from below by Max. Hence Max is the smallest disjunctive aggregation 
function. When restricting our consideration to the unit interval $\mathbb{I}=[0,1]$, there is a one-to-one connection between conjunctive and disjunctive aggregation functions (this is not true, for example, if $\mathbb{I}=] 0,1]$ or $\mathbb{I}=[0,1[$ ).

Lemma 1 Let $\varphi:[0,1] \rightarrow[0,1]$ be a decreasing bijection. Then the (extended) aggregation function $\mathrm{A}$ is disjunctive if and only if its transform $\mathrm{A}_{\varphi}$ given by

$$
\mathrm{A}_{\varphi}(\mathbf{x}):=\varphi^{-1}\left(\mathrm{~A}\left(\varphi\left(x_{1}\right), \ldots, \varphi\left(x_{n}\right)\right)\right)
$$

(see (3)) is conjunctive.

Remark 8 (i) In fuzzy logic (see [30, 35]), an involutive decreasing bijection $\varphi:[0,1] \rightarrow[0,1]$ (i.e., $\varphi(\varphi(x))=x$ for all $x \in[0,1]$ ) plays the role of negation. For any aggregation function $\mathrm{A}$ we have $\left(\mathrm{A}_{\varphi}\right)_{\varphi}=\mathrm{A}$, i.e., $\varphi$-transformation brings a kind of duality into the class of aggregation functions. Due to Lemma 1, this duality also connects the class of disjunctive aggregation functions and the class of conjunctive aggregation functions. Moreover, if a t-norm $\mathrm{T}$ models the conjunction in fuzzy logic and $\varphi$ models the negation, then the triplet $\left(\mathrm{T}, \mathrm{T}_{\varphi}, \varphi\right)$ is called a de Morgan triplet.

(ii) For $N:[0,1] \rightarrow[0,1]$ given by $N(x)=1-x$, the dual aggregation function $\mathrm{A}^{d}$ can be introduced as $\mathrm{A}^{d}=\mathrm{A}_{N}$. Thus disjunctive aggregation functions are just dual functions to conjunctive aggregation functions, and therefore we can derive all their properties from the corresponding properties of conjunctive aggregation functions. So, for example, the smallest and the only idempotent disjunctive aggregation function is Max. In fuzzy logic $N$ is called the standard negation.

As already mentioned, the only idempotent disjunctive $n$-ary (extended) aggregation is just Max, while the greatest $n$-ary (extended) aggregation function $\mathrm{A}^{\top}$ is also the greatest $n$-ary (extended) disjunctive aggregation function. As far as each idempotent aggregation function on $[0,1]$ has $a=1$ as its annihilator, it can have as a neutral element only $e=0$.

Taking into account Definition 3, the duality of conjunctive and disjunctive aggregation functions allows to introduce several classes of special disjunctive aggregation functions. To avoid superfluous repetitions, we focus here only on the case of aggregation functions dual to triangular norms, i.e., to triangular conorms .

Definition 7 The dual aggregation function to a t-norm $\mathrm{T}: \cup_{n \in \mathbb{N}}[0,1]^{n} \rightarrow$ $[0,1]$, i.e., an associative symmetric aggregation function $\mathrm{S}: \cup_{n \in \mathbb{N}}[0,1]^{n} \rightarrow[0,1]$ with extended neutral element 0 is called a triangular conorm or a t-conorm for short.

Obviously, Max is the smallest t-conorm and it is dual to the greatest t-norm Min.

By duality, t-conorms have annihilator $a=1$. For each t-conorm $\mathrm{S}$, we have $\operatorname{Max} \leqslant \mathrm{S} \leqslant \mathrm{S}_{\mathrm{D}}$, where

$$
\mathrm{S}_{\mathbf{D}}(\mathbf{x}):= \begin{cases}\operatorname{Max}(\mathbf{x}) & \text { if }\left|\left\{i \mid x_{i}>0\right\}\right|<2 \\ 1 & \text { otherwise }\end{cases}
$$


The dual function to the product $\Pi$ is called the probabilistic sum and it is denoted by $\mathrm{S}_{\mathbf{P}}$,

$$
\mathrm{S}_{\mathbf{P}}(\mathbf{x}):=1-\prod_{i=1}^{n}\left(1-x_{i}\right) .
$$

The Eukasiewicz t-conorm $\mathbf{S}_{\mathbf{L}}$ is often called the bounded sum because of

$$
\mathrm{S}_{\mathbf{L}}(\mathbf{x}):=\operatorname{Min}\left(1, \sum_{i=1}^{n} x_{i}\right)
$$

Remark 9 (i) A continuous Archimedean t-conorm $\mathrm{S}$ is characterized by the diagonal inequality $\mathrm{S}(x, x)>x$ for all $x \in] 0,1[$.

(ii)) A continuous Archimedean t-conorm $\mathrm{S}$ is always related to some continuous increasing additive generator $s:[0,1] \rightarrow[0, \infty], s(0)=0$, by

$$
\mathrm{S}(\mathbf{x})=s^{-1}\left(\operatorname{Min}\left(s(1), \sum_{i=1}^{n} s\left(x_{i}\right)\right)\right) .
$$

(iii) The t-conorms which are dual to strict $t$-norms are called strict $t$-conorms. They have unbounded additive generators, and they are isomorphic to $\mathrm{S}_{\mathbf{P}}$. Similarly nilpotent t-conorms are dual to nilpotent $t$-norms (with bounded additive generators, isomorphic to $\mathrm{S}_{\mathbf{L}}$ ). The duality of continuous Archimedean t-norms and t-conorms is reflected by the duality $s=t \circ N$ (where $N$ is the standard negation, $N(x)=1-x$ ) of the corresponding additive generators, i.e., $s(u)=t(1-u), u \in[0,1]$. Consequently, $s_{\mathbf{P}}(u)=-\log (1-u)$ generates the probabilistic sum $\mathbf{S}_{\mathbf{P}}$, while $s_{\mathbf{L}}(u)=u$ generates the bounded sum $\mathrm{S}_{\mathbf{L}}$.

(iv) The representation of continuous t-norms in Therem 2(iv) is reflected by the dual representation of continuous t-conorms,

$$
\mathrm{S}(\mathbf{x})= \begin{cases}s_{k}^{-1}\left(\operatorname{Min}\left(s_{k}\left(b_{k}\right), \sum_{i=1}^{n} s_{k}\left(\operatorname{Max}\left(x_{i}, a_{k}\right)\right)\right)\right) & \text { if } \operatorname{Max}(\mathbf{x}) \in] a_{k}, b_{k}[ \\ \operatorname{Max}(\mathbf{x}) & \text { otherwise, }\end{cases}
$$

where (]$a_{k}, b_{k}[)_{k \in K}$ is a family of pairwise disjoint subintervals of $[0,1]$, and $s_{k}:\left[a_{k}, b_{k}\right] \rightarrow[0, \infty], s_{k}\left(a_{k}\right)=0$, is a corresponding family of continuous increasing mappings. A continuous t-conorm $\mathrm{S}$ as given in (26) is, in general, a disjunctive ordinal sum of disjunctive aggregation functions, and each of classes of disjunctive aggregation functions which can be introduced by means of duality and Definition 3 is closed under this type of ordinal sums.

In several domains with inputs from the interval $\mathbb{I}=[e, a]$, a special kind of disjunctive aggregation functions called pseudo-additions is required. Due to the associativity of a pseudo-addition, only its binary form is now introduced.

Definition 8 Let $\mathbb{I}=[a, b]$ be fixed. A mapping $\oplus: \mathbb{I}^{2} \rightarrow \mathbb{I}$ is called a pseudoaddition (on $\mathbb{I})$ if it satisfies the next properties: 
(i) associativity, i.e., $(x \oplus y) \oplus z=x \oplus(y \oplus z)$ for all $x, y, z \in \mathbb{I}$;

(ii) monotonicity, i.e., $x \oplus y \leqslant x^{\prime} \oplus y^{\prime}$ for all $x, y, x^{\prime}, y^{\prime} \in \mathbb{I}, x \leqslant x^{\prime}$ and $y \leqslant y^{\prime}$;

(iii) $a$ is the neutral element of $\oplus$, i.e., $x \oplus a=a \oplus x=x$ for all $x \in \mathbb{I}$;

(iv) continuity.

Note that based on the classical result of Mostert and Shields [53], each pseudoaddition is an $\mathbb{I}$-semigroup and thus the symmetry of $\oplus$ follows. Moreover, [53] provides also a representation theorem for pseudo-additions. It is not difficult to see that in the special case $\mathbb{I}=[0,1]$, pseudo-additions are just continuous t-conorms, and then the representation theorem from [53] is just the disjunctive ordinal sum given in (26). In the theory of non-additive integrals $[57,58,65]$, pseudo-additions on $\mathbb{I}=[0, \infty]$ play an important role.

Proposition 18 A mapping $\oplus:[0, \infty]^{2} \rightarrow[0, \infty]$ is a pseudo-addition on $[0, \infty]$ if and only if there is a family (]$a_{k}, b_{k}[)_{k \in K}$ of pairwise disjoint subintervals of $] 0, \infty\left[\right.$, and $f_{k}:\left[a_{k}, b_{k}\right] \rightarrow[0, \infty], f_{k}\left(a_{k}\right)=0$, is a corresponding family of continuous strictly increasing functions, so that

$$
x \oplus y= \begin{cases}f_{k}^{-1}\left(\min \left(f_{k}\left(b_{k}\right), f_{k}(x)+f_{k}(y)\right)\right) & \text { if }(x, y) \in] a_{k}, b_{k}\left[^{2},\right. \\ \max (x, y) & \text { otherwise. }\end{cases}
$$

Note that the standard addition + on $[0, \infty]$ corresponds to $K=\{1\}, a_{1}=$ $0, b_{1}=\infty$, and $f_{1}(x)=x$ for $x \in[0, \infty]$, while $K=\varnothing$ is linked with the idempotent pseudo-addition $\oplus=$ Max.

As already mentioned, triangular norms and triangular conorms model in fuzzy set theory the intersection and the union of fuzzy sets, respectively. The classical valuation property of characteristic functions of standard sets, i.e., the validity of

$$
\mathbf{1}_{A \cup B}+\mathbf{1}_{A \cap B}=\mathbf{1}_{A}+\mathbf{1}_{B}
$$

for any two subsets $A, B$ of a given universe $X$, is then reflected in the fuzzy set theory by the famous Frank functional equation [23]

$$
\mathrm{T}(x, y)+\mathrm{S}(x, y)=x+y \text { for all } x, y \in[0,1]
$$

where $\mathrm{T}$ is a t-norm and $\mathrm{S}$ is a t-conorm (note that the original motivation of Frank in [23] was related to associative copulas and associative dual copulas). Evidently, $\mathrm{T}=\mathrm{Min}$ and $\mathrm{S}=\mathrm{Max}$ is a trivial solution of (27). Moreover, also $\mathrm{T}_{P}=\Pi$ with $S_{P}$, and $\mathrm{T}_{L}$ with $\mathrm{S}_{L}$ solve [23]. The solution of (27) in the class of triangular norms with no non-trivial idempotent elements gave birth to the wellknown parametric class $\left(\mathrm{T}_{\lambda}^{F}\right)_{\lambda \in[0, \infty]}$ of Frank t-norms, where $\mathrm{T}_{0}^{F}=\operatorname{Min}, \mathrm{T}_{1}^{F}=$ $\Pi, \mathrm{T}_{\infty}^{F}=\mathrm{T}_{\mathbf{L}}$, and for $\left.\lambda \in\right] 0,1[\cup] 1, \infty[$ it holds

$$
\mathrm{T}_{\lambda}^{F}(x, y)=\log _{\lambda} \frac{1+\left(\lambda^{x}-1\right)\left(\lambda^{y}-1\right)}{\lambda-1} .
$$

Note that for $\lambda \in] 0, \infty[$, all Frank t-norms are strict, and for $\lambda \in[0, \infty]$, all Frank t-norms are also binary copulas (and thus, they are 1-Lipschitz conjunctors). Frank t-conorms $S_{\lambda}^{F}$ have two equivalent expressions, namely, for all $x, y \in[0,1]$ it holds

$$
\mathrm{S}_{\lambda}^{F}(x, y)=x+y-\mathrm{T}_{\lambda}^{F}(x, y)=1-\mathrm{T}_{\lambda}^{F}(1-x, 1-y) .
$$


Up to the prominent role of Frank t-norms and t-conorms as fuzzy connectives in the fuzzy set theory, observe that there are important applications of Frank t-norms and t-conorms in fuzzy game theory, see [8].

\section{$5 \quad$ Mixed aggregation functions}

There are several aggregation functions which are neither averaging, nor conjunctive or disjunctive. As a typical example recall the standard summation on the real line, or the standard product on $[0, \infty]$. Aggregation functions of this type will be called mixed. On some sub-domains, mixed aggregation functions behave like averaging, or conjunctive, or disjunctive aggregation functions. For example, considering the product $\Pi:[0, \infty]^{2} \rightarrow[0, \infty]$, it is conjunctive on $[0,1]^{2}$, disjunctive on $[1, \infty]^{2}$ and averaging of the remainder of its domain. In this section, we will discuss some well-known classes of mixed aggregation functions. Maybe the most prominent class of this type is formed by uninorms introduced by Yager and Rybalov in [75], see also [18, 34, 57].

Definition 9 An aggregation function $\mathrm{U}: \cup_{n \in \mathbb{N}}[0,1]^{n} \rightarrow[0,1]$ which is symmetric, associative and possesses an extended neutral element $e \in] 0,1[$ is called a uninorm.

Observe that each uninorm $\mathrm{U}$ has an annihilator $a=\mathrm{U}(0,1) \in\{0,1\}$. If $a=0$, then the uninorm $\mathrm{U}$ is called conjunctive, as it can be seen as an extension of the Boolean conjunction. Similarly, disjunctive uninorms are characterized by annihilator $a=1$. We should stress that there is no continuous uninorm! A similar problem we see in the case of the standard product $\Pi$ on $[0, \infty]$, where the points $(0, \infty)$ and $(\infty, 0)$ are points of discontinuity, independently of the convention determining the value of $\Pi(0, \infty)$. A typical example of a conjunctive uninorm (with the only discontinuity points $(0,1)$ and $(1,0)$ ) is the 3 - $П$-operator $\mathrm{E}$, see [74], given by

$$
\mathrm{E}(\mathbf{x})=\frac{\prod_{i=1}^{n} x_{i}}{\prod_{i=1}^{n} x_{i}+\prod_{i=1}^{n}\left(1-x_{i}\right)} \text {, with the convention } \frac{0}{0}=0 .
$$

The structure of uninorms is strongly connected with t-norms and t-conorms. For a given uninorm $\mathrm{U}$ with a neutral element $e$, we introduce the related t-norm $\mathrm{T}_{\mathrm{U}}:[0, e]^{2} \rightarrow[0, e]$ and the t-conorm $\mathrm{S}_{\mathrm{U}}:[e, 1]^{2} \rightarrow[e, 1]$, given by

$$
\mathrm{T}_{\mathrm{U}}(x, y):=\frac{\mathrm{U}(e x, e y)}{e}
$$

and

$$
\mathrm{S}_{\mathrm{U}}(x, y):=\frac{\mathrm{U}(e+(1-e) x, e+(1-e) y)-e}{1-e} .
$$

We characterize the binary form of a uninorm $U$, which due to the associativity of $\mathrm{U}$, gives complete information about $\mathrm{U}$.

Proposition 19 Let $\mathrm{U}:[0,1]^{2} \rightarrow[0,1]$ be a uninorm with neutral element e $\in$ ] $0,1\left[\right.$. Then there are three binary aggregation functions $\mathrm{T}, \mathrm{S}, \mathrm{H}:[0,1]^{2} \rightarrow[0,1]$ 
such that $\mathrm{T}$ is a t-norm, $\mathrm{S}$ a t-conorm and $\mathrm{H}$ is a symmetric mean aggregation function (see [29], Definition 25), and for any $\mathbf{x} \in[0,1]^{2}$ we have (see Figure 2)

$$
\mathrm{U}(\mathbf{x})= \begin{cases}\mathrm{T}(\mathbf{x}) & \text { if } \mathbf{x} \in[0, e]^{2} \\ \mathrm{~S}(\mathbf{x}) & \text { if } \mathbf{x} \in[e, 1]^{2} \\ \mathrm{H}(\mathbf{x}) & \text { otherwise }\end{cases}
$$

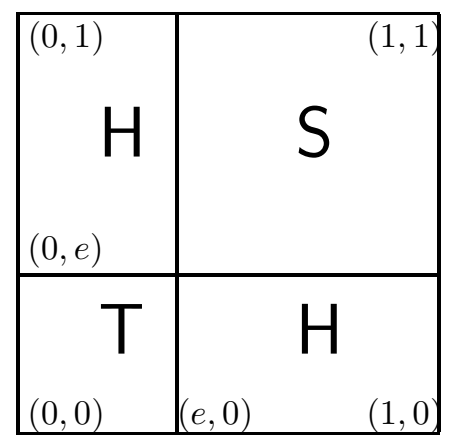

Figure 2: The representation of a uninorm from Proposition 19

Proposition 19 gives the necessary but not sufficient representation of (binary) uninorms by means of t-norms, t-conorms and symmetric mean functions. For a given t-norm $T_{\mathrm{U}}$, t-conorm $\mathrm{S}_{\mathrm{U}}$ and neutral element $\left.e \in\right] 0,1[$, to find an appropriate uninorm $\mathrm{U}$ means to find a symmetric mean aggregation function $\mathrm{H}$ so that (30) holds, where $\left.\mathrm{T}=\left(<0, e, \mathrm{~T}_{\mathbf{U}}\right\rangle\right)$ and $\left.\mathbf{S}=\left(<e, 1, \mathrm{~S}_{\mathbf{U}}\right\rangle\right)$. The main problem is to ensure the associativity of the function $\mathrm{U}$ constructed in such a way.

Proposition 20 Let $e \in] 0,1\left[\right.$ be a given constant, and let $\left.\mathrm{T}=\left(<0, e, \mathrm{~T}_{\mathrm{U}}\right\rangle\right)$ and $\left.\mathbf{S}=\left(<e, 1, \mathrm{~S}_{\mathrm{U}}\right\rangle\right)$ be an ordinal sum of t-norms and an ordinal sum of $t$-conorms, respectively. Then the following holds.

(i) For any uninorm $\mathrm{U}$ characterized by $e, \mathrm{~T}_{\mathrm{U}}$ and $\mathrm{S}_{\mathrm{U}}$, we have

$$
\mathrm{T}<\mathrm{U}_{e, \mathrm{~T}, \mathrm{~S}} \leqslant \mathrm{U} \leqslant \mathrm{U}_{\mathrm{T}, \mathrm{S}, e}<\mathrm{S},
$$

where

$$
\mathrm{U}_{e, \mathrm{~T}, \mathrm{~S}}(x, y):= \begin{cases}e \mathrm{~T}\left(\frac{x}{e}, \frac{y}{e}\right) & \text { if }(x, y) \in[0, e]^{2}, \\ e+(1-e) \mathrm{S}\left(\frac{x-e}{1-e}, \frac{y-e}{1-e}\right) & \text { if }(x, y) \in[e, 1]^{2}, \\ \operatorname{Min}(x, y) & \text { otherwise, }\end{cases}
$$

and

$$
\mathrm{U}_{\mathrm{T}, \mathrm{S}, e}(x, y):= \begin{cases}e \mathrm{~T}\left(\frac{x}{e}, \frac{y}{e}\right) & \text { if }(x, y) \in[0, e]^{2}, \\ e+(1-e) \mathrm{S}\left(\frac{x-e}{1-e}, \frac{y-e}{1-e}\right) & \text { if }(x, y) \in[e, 1]^{2}, \\ \operatorname{Max}(x, y) & \text { otherwise. }\end{cases}
$$


(ii) $\mathrm{U}_{e, \mathrm{~T}, \mathrm{~S}}$ and $\mathrm{U}_{\mathrm{T}, \mathrm{S}, e}$ are uninorms.

Note that uninorms need not be mixed aggregation functions, in general. Indeed, some of uninorms are idempotent, see e.g. [17], and thus they are averaging aggregation functions.

Example 8 Let $\varphi:[0,1] \rightarrow[0,1]$ be an increasing bijection and define $\mathrm{U}^{(\varphi)}$ : $[0,1]^{2} \rightarrow[0,1]$ by

$$
\mathrm{U}^{(\varphi)}(x, y):= \begin{cases}\operatorname{Min}(x, y) & \text { if } \varphi(x)+\varphi(y) \leqslant 1 \\ \operatorname{Max}(x, y) & \text { otherwise. }\end{cases}
$$

Then $\mathrm{U}^{(\varphi)}$ is a (left-continuous, conjunctive) uninorm with neutral element $e=$ $\varphi^{-1}(0.5)$.

Another important class of uninorms is formed by so called representable uninorms $[22,34]$ which are isomorphic to the product $\Pi$ on $[0, \infty]$ (or, equivalently, to the sum $\Sigma$ on $[-\infty, \infty]$ ). Based on Aczél's representation theorem [1] for continuous cancellative associative functions, the next result can be shown.

Theorem 7 A function $\mathrm{U}: \cup_{n \in \mathbb{N}}[0,1]^{n} \rightarrow[0,1]$ is a uninorm continuous and cancellative on $\left.\cup_{n \in \mathbb{N}}\right] 0,1{ }^{n}$ if and only if there exists a monotone bijection $u$ : $[0,1] \rightarrow[-\infty, \infty]$ such that

$$
\mathrm{U}(\mathbf{x})=u^{-1}\left(\sum_{i=1}^{n} u\left(x_{i}\right)\right),
$$

with convention $\infty+(-\infty)=-\infty$. The uninorm $\mathrm{U}$ is then called a representable uninorm with additive generator $u$, and an additive generator $u$ is unique up to a positive multiplicative constant.

Obviously, a multiplicative version of (34) exist, i.e.,

$$
\mathrm{U}(\mathbf{x})=v^{-1}\left(\Pi_{i=1}^{n} v\left(x_{i}\right)\right),
$$

where $v:[0,1] \rightarrow[0, \infty]$ is a monotone bijection (unique up to a positive power constant!) and the convention $\Pi(0, \infty)=0$ is adopted. Observe that a representable uninorm $\mathrm{U}$ with an additive generator $u$ (multiplicative generator $v$ ) is conjunctive if and only if $u(v)$ is increasing. Similarly, disjunctive representable uninorms are characterized by decreasing generators. 3-П-operator $\mathrm{E}$ introduced above is a conjunctive representable uninorm with an additive generator $u:[0,1] \rightarrow[-\infty, \infty]$ given by $u(x)=\log \left(\frac{x}{1-x}\right)$, and with a multiplicative generator $v:[0,1] \rightarrow[0, \infty]$ given by $v(x)=\frac{x}{1-x}$. The class of uninorms is closed under duality. Note only that a dual to a conjunctive uninorm with a neutral element $e$ is a disjunctive uninorm with a neutral element $1-e$. For more details we recommend [28], Section 3.6.

Each non-idempotent uninorm is an example of a mixed aggregation function on an interval $\mathbb{I}$ possessing a neutral element $e$ which is an interior point of $\mathbb{I}$. The structure of such mixed aggregation functions is closely related to the structure of uninorms described in Proposition 19. 
Proposition 21 Let $\mathrm{B}:[0,1]^{2} \rightarrow[0,1]$ be a non-idempotent aggregation function with neutral element $e \in] 0,1[$. Then $\mathrm{B}$ is a mixed aggregation function and there are three binary aggregation functions $\mathrm{C}, \mathrm{D}, \mathrm{A}:[0,1]^{2} \rightarrow[0,1]$ such that $\mathrm{C}$ is conjunctive, $\mathrm{D}$ is disjunctive and $\mathrm{A}$ is an averaging aggregation function, and for any $(x, y) \in[0,1]^{2}$ we have

$$
\mathrm{B}(x, y)= \begin{cases}\mathrm{C}(x, y) & \text { if }(x, y) \in[0, e]^{2}, \\ \mathrm{D}(x, y) & \text { if }(x, y) \in[e, 1]^{2}, \\ \mathrm{~A}(x, y) & \text { otherwise. }\end{cases}
$$

Example 9 Fix a constant $e \in] 0,1\left[\right.$ and let $\mathrm{B}:[0,1]^{2} \rightarrow[0,1]$ be given by

$$
\mathrm{B}(x, y)=\operatorname{Max}(0, \operatorname{Min}(1, x+y-e)) .
$$

Then $\mathrm{B}$ is a symmetric mixed aggregation function with a neutral element $e$. Observe that $\mathrm{B}$ is continuous and hence it cannot be a uninorm (indeed, $\mathrm{B}$ is not associative). $\mathrm{B}$ possesses a representation (35), where $\mathrm{C}=\left(\left\langle 0, e, \mathrm{~T}_{\mathbf{L}}\right\rangle\right)$ is an ordinal sum $t$-norm and $\left.\mathrm{D}=\left(<e, 1, \mathrm{~S}_{\mathbf{L}}\right\rangle\right)$ is an ordinal sum $t$-conorm.

As already seen in the case of uninorms, additional properties posed on mixed aggregation functions with a neutral element $e \in] 0,1[$ have impact on the characterization of related conjunctive operators $C$ and disjunctive operators $D$. One of such properties is 1-Lipschitzianity, see [39].

Proposition 22 Let $\mathrm{B}:[0,1]^{2} \rightarrow[0,1]$ be a 1-Lipschitz function. Then $\mathrm{B}$ is a mixed aggregation function with a neutral element $e \in] 0,1[$ if and only if there are quasi-copulas $\mathrm{C}, \mathrm{Q}:[0,1]^{2} \rightarrow[0,1]$ so that

$$
\mathrm{B}(x, y)= \begin{cases}e \mathrm{C}\left(\frac{x}{e}, \frac{y}{e}\right) & \text { if }(x, y) \in[0, e]^{2}, \\ 1-(1-e) \mathrm{Q}\left(\frac{1-x}{1-e}, \frac{1-y}{1-e}\right) & \text { if }(x, y) \in[e, 1]^{2}, \\ x+y-e & \text { otherwise. }\end{cases}
$$

Observe that the function $B$ from Example 9 satisfies the requirements of Proposition 22 , with $C=Q=T_{L}$.

When considering aggregation functions on an interval $\mathbb{I}$, also the position of the corresponding annihilator $a$ (if it exists) has the impact on the classification of the discussed aggregation function. Clearly, for $\mathbb{I}=[0,1], a<1$ excludes disjunctive aggregation functions. Similarly, $a>0$ excludes conjunctive aggregation functions. Therefore, $a \in] 0,1[$ admits mixed or averaging aggregation functions only.

Proposition 23 Let $\mathrm{B}:[0,1]^{2} \rightarrow[0,1]$ be a non-idempotent aggregation function with annihilator $a \in] 0,1[$. Then $\mathrm{B}$ is a mixed aggregation function and there are two binary aggregation functions $\mathrm{C}, \mathrm{D}:[0,1]^{2} \rightarrow[0,1]$ such that $\mathrm{C}$ is conjunctive, $\mathrm{D}$ is disjunctive, and for any $(x, y) \in[0,1]^{2}$ we have

$$
\mathrm{B}(x, y)= \begin{cases}\mathrm{D}(x, y) & \text { if }(x, y) \in\left[0, a\left[^{2},\right.\right. \\ \mathrm{C}(x, y) & \text { if }(x, y) \in] a, 1]^{2}, \\ a & \text { otherwise. }\end{cases}
$$


Evidently, B given in (36) is symmetric whenever both C and D are symmetric. Moreover, also the associativity of $\mathrm{C}$ and $\mathrm{D}$ ensures the associativity of $\mathrm{B}$.

We have by [11] the following definition.

Definition 10 A symmetric associative aggregation function $\mathrm{V}:[0,1]^{2} \rightarrow[0,1]$ with an annihilator $a \in] 0,1[$ is called a nullnorm.

Idempotent nullnorms are completely characterized by their annihilator $a$ and they are known as $a$-medians $([21,25]) \operatorname{Med}_{a}:[0,1]^{2} \rightarrow[0,1]$, given by $\operatorname{Med}_{a}(x, y)=$ $\operatorname{Med}(x, a, y)$. As observed in [12], nullnorms are closely related to t-norms, tconorms and $a$-medians.

Proposition 24 A function $\mathrm{V}:[0,1]^{2} \rightarrow[0,1]$ is a nullnorm with an annihilator $a \in] 0,1\left[\right.$ if and only if there is a t-norm $\mathrm{T}:[0,1]^{2} \rightarrow[0,1]$ and a $t$-conorm $\mathrm{S}:[0,1]^{2} \rightarrow[0,1]$ such that for any $(x, y) \in[0,1]^{2}$ it holds $\mathrm{V}(x, y)=$ $\operatorname{Med}_{a}(\mathrm{~T}(x, y), \mathbf{S}(x, y))$.

Example 10 Fix $a=0.5, \mathrm{~T}=\Pi=\mathrm{T}_{\mathbf{P}}$ and $\mathrm{S}=\mathrm{S}_{\mathbf{P}}$. Then the nullnorm $\mathrm{V}$ characterized by the above proposition is given by

$$
\mathrm{V}(x, y)= \begin{cases}x y & \text { if } x y>0.5 \\ x+y-x y & \text { if }(1-x)(1-y)>0.5 \\ 0.5 & \text { otherwise. }\end{cases}
$$

There are several other mixed aggregation functions related to triangular norms and triangular conorms.

The gamma operators $\Gamma_{\gamma}: \cup_{n \in \mathbb{N}}[0,1]^{n} \rightarrow[0,1]$ were introduced by Zimmermann and Zysno [77] and applied to car control. For a parameter $\gamma \in[0,1]$, the gamma operator $\Gamma_{\gamma}$ is given by $\Gamma_{\gamma}:=\Pi^{1-\gamma} S_{\mathbf{P}}^{\gamma}$, that is,

$$
\Gamma_{\gamma}(\mathbf{x}):=\left(\prod_{i=1}^{n} x_{i}\right)^{1-\gamma}\left(1-\prod_{i=1}^{n}\left(1-x_{i}\right)\right)^{\gamma} .
$$

Gamma operators can be seen as a weighted geometric mean applied to the product $\Pi$ and the probabilistic sum $S_{\mathbf{P}}$. Though they are not associative (up to the boundary cases $\gamma=0$ or $\gamma=1$ ), their components $\Pi$ and $\mathbf{S}_{\mathbf{P}}$ are associative, what can be exploited by applications of these operators.

Gamma operators are a special subclass of the so called exponential convex T-S-operators [40], that is, of weighted geometric means of a t-norm $\mathrm{T}$, and a t-conorm $\mathrm{S}$ (not necessarily a dual pair), $\mathrm{E}_{\mathrm{T}, \mathrm{S}, \gamma}: \cup_{n \in \mathbb{N}}[0,1]^{n} \rightarrow[0,1]$,

$$
\mathrm{E}_{\mathrm{T}, \mathrm{S}, \gamma}(\mathbf{x}):=(\mathrm{T}(\mathbf{x}))^{1-\gamma}(\mathrm{S}(\mathbf{x}))^{\gamma} .
$$

Another composed aggregation approach based on t-norms and t-conorms is related to the weighted arithmetic mean (as the outer function) [40]. A linear convex T-S-operator $\mathrm{L}_{\mathrm{T}, \mathrm{S}, \gamma}: \cup_{n \in \mathbb{N}}[0,1]^{n} \rightarrow[0,1]$ is given by

$$
\mathrm{L}_{\mathrm{T}, \mathrm{S}, \gamma}(\mathbf{x}):=(1-\gamma) \mathrm{T}(\mathbf{x})+\gamma \mathbf{S}(\mathbf{x}) .
$$

These operators were successfully applied in fuzzy linear programming. Linear convex T-S-operators are symmetric, continuous whenever $\mathrm{T}$ and $\mathrm{S}$ are continuous, neither with annihilator nor with neutral element whenever $\gamma \in] 0,1[$. 
Example 11 Consider the following linear convex operator

$$
L_{\mathbf{T}_{\mathbf{P}}, \mathrm{S}_{\mathbf{P}}, 0.4}(x, y)=0.6 \cdot \mathrm{T}_{\mathbf{L}}(x, y)+0.4 \cdot \mathrm{S}_{\mathbf{P}}(x, y)=0.4 x+0.4 y+0.2 x y .
$$

Obviously, the above two concepts can be further generalized into Exponential convex C-D-operators and into Linear convex C-D-operators with C a conjunctive and $\mathrm{D}$ a disjunctive aggregation function. Moreover, as an outer composition we can replace the geometric mean (applied in the case of exponential convex operators) and the weighted arithmetic mean (applied in the case of linear convex operators) by any other averaging binary aggregation function.

Example 12 Consider the harmonic mean $\operatorname{HM}(x, y)=\frac{2 x y}{x+y}$, the product $\Pi$ and the probabilistic sum $\mathbf{S}_{\mathbf{P}}$. Then the corresponding mixed aggregation function $\mathrm{B}:[0,1]^{2} \rightarrow[0,1]$ is given by

$$
\mathrm{B}(x, y)=\mathrm{HM}\left(\Pi(x, y), \mathbf{S}_{\mathbf{P}}(x, y)\right)=\frac{2 x y(x+y-x y)}{x+y}
$$

whenever $(x, y) \neq(0,0)$. Evidently, $\mathrm{B}$ is not averaging (it is not idempotent). Moreover, $\mathrm{B}(0.1,0.1)=0.019<0.1$ and thus $\mathrm{B}$ cannot be disjunctive. Similarly, $\mathrm{B}(1,0.5)=2 / 3>0.5$ and thus $\mathrm{B}$ cannot be conjunctive. Thus $\mathrm{B}$ is a mixed aggregation function.

\section{Concluding remarks}

In our two-part contribution we have introduced and discussed the basics of the theory of aggregation functions. Besides several properties and construction methods, also several kinds of aggregation functions have been introduced and examined. The application of aggregation functions can be found in any domain where the observed pieces of information are merged into a single value. We indicate some of the domains where aggregation functions play a substantial role.

We do not pretend to be exhaustive, and the reader may consult more application-oriented books on aggregation, e.g., [67].

A first group of applications comes from decision theory. Making decisions often amounts to aggregating scores or preferences on a given set of alternatives, the scores or preferences being obtained from several decision makers, voters, experts, etc., or represent different points of view, criteria, objectives, etc. This concerns decision under multiple criteria or multiple attributes, multiperson decision making, and multiobjective optimization.

A second group is rooted in information or data fusion. The aim is to refine the information on a given set of objects, by fusing several sources. Often, this amounts to making some kind of decision, as in the first group of applications. Typical applications here are pattern recognition and classification, as well as image analysis.

A third group comes from artificial intelligence and fuzzy logic. Aggregation functions are essentially used there as a generalization of logical connectives in rule-based systems (automated reasoning).

Finally, we recall the probability theory and risk management with applications in hydrology, finance, etc., where copulas play a prominent role. To 
stress the expansive development of aggregation theory and its applications in information sciences we recall some of recent publications in this area, namely $[7,9,24,43,45,47,59,69,70]$.

\section{Acknowledgment}

The authors gratefully acknowledge the support of following projects: bilateral project between Slovakia and Serbia SK-SRB-19, the internal research project supported by the University of Luxembourg "Mathematics Research in Decision Making and Operations Research", F1R-MTH-PUL-09MRDO,, project APVV0012-07 supported by the Slovak grant agency, the national grants Ministry of Sciences of Serbia 174009 (Mathematical models of nonlinearity, uncertainty and decision), Provincial Secretariat for Science and Technological Development of Vojvodina. The article has drawn heavily upon material from Michel Grabisch, Jean-Luc Marichal, Radko Mesiar, Endre Pap, Aggregation Functions, (C)M. Grabisch, J.-L. Marichal, R. Mesiar and E. Pap 2009, published by Cambridge University Press, extracts reproduced with permission.

\section{References}

[1] J. Aczél. Lectures on functional equations and their applications. Mathematics in Science and Engineering, Vol. 19. Academic Press, New York, 1966. Translated by Scripta Technica, Inc. Supplemented by the author. Edited by Hansjorg Oser.

[2] J. Aczél and C. Alsina. Characterizations of some classes of quasilinear functions with applications to triangular norms and to synthesizing judgments. Methods Oper. Res., 48:3-22, 1984.

[3] C. Alsina, M. J. Frank, and B. Schweizer. Associative functions, Triangular norms and copulas. World Scientific Publishing Co. Pte. Ltd., Hackensack, NJ, 2006.

[4] C. Alsina, R. B. Nelsen, and B. Schweizer. On the characterization of a class of binary operations on distribution functions. Statist. Probab. Lett., 17(2):85-89, 1993.

[5] M. Bajraktarević. Sur une équation fonctionnelle aux valeurs moyennes. Glasnik Mat.-Fiz. Astronom. Društvo Mat. Fiz. Hrvatske. Ser. II, 13:243$248,1958$.

[6] G. Birkhoff. Lattice theory. Third edition. American Mathematical Society Colloquium Publications, Vol. XXV. American Mathematical Society, Providence, R.I., 1967.

[7] H. Bustince, T. Calvo, B. De Baets, J. Fodor, R. Mesiar, J. Montero, D. paternan, and A. Pradera. A class of aggregation functions encompassing two-dimensional OWA operators. Inform. Sci., 180(10):1977-1989, 2010.

[8] D. Butnariu and E. P. Klement. Triangular norm-based measures and games with fuzzy coalitions. Kluwer Academic Publishers, 1993. 
[9] G. Büyüközkan and D. Ruan. Choquet integral based aggregation approach to software development risk assessment. Inform. Sci., 180(3):441-451, 2010 .

[10] T. Calvo and G. Beliakov. Aggregation functions based on penalties. Fuzzy Sets and Systems, 161(10):1420-1436, 2010.

[11] T. Calvo, B. De Baets, and J. Fodor. The functional equations of Frank and Alsina for uninorms and nullnorms. Fuzzy Sets and Systems, 120(3):385$394,2001$.

[12] T. Calvo, A. Kolesárová, M. Komorníková, and R. Mesiar. Aggregation operators: properties, classes and construction methods. In Aggregation operators: new trends and applications, pages 3-104. Physica, Heidelberg, 2002.

[13] T. Calvo, R. Mesiar, and R. R. Yager. Quantitative weights and aggregation. IEEE Trans. Fuzzy and Systems, 12(1):62-69, 2004.

[14] T. Calvo and A. Pradera. Double aggregation operators. Fuzzy Sets and Systems, 142(1):15-33, 2004.

[15] P. Capéraà, A.-L. Fougères, and C. Genest. Bivariate distributions with given extreme value attractor. J. Multivariate Anal., 72(1):30-49, 2000.

[16] I. Cuculescu and R. Theodorescu. Extreme value attractors for star unimodal copulas. C. R. Math. Acad. Sci. Paris, 334(8):689-692, 2002.

[17] B. De Baets and J. Fodor. Van Melle's combibing function in MYCIN is a representable uninorm: an alternative proof. Fuzzy Sets and Systems, 104(1):133-136, 1999.

[18] J. Dombi. Basic concepts for a theory of evaluation: The aggregative operator. Eur. J. Oper. Res., 10:282-293, 1982.

[19] F. Durante and C. Sempi. Semicopulæ. Kybernetika (Prague), 41(3):315$328,2005$.

[20] J. C. Fodor. Contrapositive symmetry of fuzzy implications. Fuzzy Sets and Systems, 69(2):141-156, 1995.

[21] J. C. Fodor. An extension of Fung-Fu's theorem. Intern. J. Uncertain. Fuzziness Knowledge-Based Systems, 4(3):235-243, 1996.

[22] J. C. Fodor, R. R. Yager, and A. Rybalov. Structure of uninorms. Int. J. Uncertain. Fuzziness Knowledge-Based Systems, 5(4):411-427, 1997.

[23] M. J. Frank. On the simultaneous associativity of $F(x, y)$ and $x+y-$ $F(x, y)$. Aequationes Math., 19(2-3):194-226, 1979.

[24] J. Fu, H. J. Caulfield, S.-M. Yoo, and D. Wu. Fuzzy aggregation with artificial color filters. Inform. Sci., 180(1):167-180, 2010. 
[25] L. W. Fung and K. S. Fu. An axiomatic approach to rational decision making in a fuzzy environment. In Fuzzy sets and their applications to cognitive and decision processes (Proc. U. S.-Japan Sem., Univ. Calif., Berkeley, Calif., 1974), pages 227-256. Academic Press, New York, 1975.

[26] J. Galambos. The asymptotic theory of extreme order statistics. Robert E. Krieger Publishing Co. Inc., Melbourne, FL, second edition, 1987.

[27] C. Genest, J. J. Quesada Molina, J. A. Rodríguez Lallena, and C. Sempi. A characterization of quasi-copulas. J. Multivariate Anal., 69(2):193-205, 1999 .

[28] M. Grabisch, J.-L. Marichal, R. Mesiar, and E. Pap. Aggregation Functions. Encyclopedia of Mathematics and its Applications 127. Cambridge University Press, 2009.

[29] M. Grabisch, J.-L. Marichal, R. Mesiar, and E. Pap. Aggregation Functions. Means. Inform. Sci., 2010.

[30] P. Hájek. Metamathematics of Fuzzy Logic. Kluwer Academic Publishers, Dordrecht, 1998.

[31] H. Hamacher. Über logische Aggregationen nicht-binär explizierter Entscheidungskriterien. Rita G. Fischer Verlag, 1978.

[32] S. Jenei. Structure of left-continuous triangular norms with strong induced negations. II. Rotation-annihilation construction. J. Appl. Non-Classical Logics, 11(3-4):351-366, 2001.

[33] H. Joe. Multivariate models and dependence concepts, volume 73 of Monographs on Statistics and Applied Probability. Chapman \& Hall, London, 1997.

[34] E. P. Klement, R. Mesiar, and E. Pap. On the relationship of associative compensatory operators to triangular norms and conorms. Int. J. of Uncertainty, Fuzziness and Knowledge-Based Systems, 4:129-144, 1996.

[35] E. P. Klement, R. Mesiar, and E. Pap. Triangular norms, volume 8 of Trends in Logic - Studia Logica Library. Kluwer Academic Publishers, Dordrecht, 2000.

[36] E. P. Klement, R. Mesiar, and E. Pap. Uniform approximation of associative copulas by strict and non-strict copulas. Illinois J. Math., 45(4):13931400, 2001.

[37] E. P. Klement, R. Mesiar, and E. Pap. Archimax copulas and invariance under transformations. C. R. Math. Acad. Sci. Paris, 340(10):755-758, 2005 .

[38] E. P. Klement, R. Mesiar, and E. Pap. Transformations of copulas. Kybernetika (Prague), 41(4):425-434, 2005.

[39] A. Kolesárová and M. Komorníková. Triangular norm-based iterative compensatory operators. Fuzzy Sets and Systems, 104(1):109-120, 1999. 
[40] M. K. Luhandjula. Compensatory operators in fuzzy linear programming with multiple objectives. Fuzzy Sets and Systems, 8(3):245-252, 1982.

[41] X. Luo and N. R. Jennings. A spectrum of compromise aggregation operators for multi-attribute decision making. Artificial Intelligence, 171(23):161-184, 2007.

[42] R. A. Marques Pereira and R. A. Ribeiro. Aggregation with generalized mixture operators using weighting functions. Fuzzy Sets and Systems, 137(1):43-58, 2003. Preference modelling and applications (Granada, 2001).

[43] G. Mayor and O. Valero. Aggregation of asymmetric distances in computer science. Inform. Sci., 180:6:803-812, 2010.

[44] A. J. McNeil and J. Nešlehová. Multivariate archimedean copulas, dmonotone functions and $l_{1}$-norm symmetric distributions. The Annals of Statistics, 37:3059-3097, 2009.

[45] J. M. Merigó and A. M. Gil-Lafuente. New decision-making techniques and their application in the selection of financial products. Inform. Sci., 180(11):2085-2094, 2010.

[46] R. Mesiar. Generated conjunctors and related operators in mv-logic as a basis for ai applications. In ECAI'98, Workshop 17, pages 1-5, Brighton, 1998.

[47] R. Mesiar, H. Bustince, and J. Fernández. On the alpha-migrativity of semicopulas, quasi-copulas, and copulas. Inform. Sci., 180(10):1967-1976, 2010.

[48] R. Mesiar and J. Špirková. Weighted means and weighting functions. Kybernetika (Prague), 42(2):151-160, 2006.

[49] R. Mesiar and J. Špirková. Weighted means and weighting functions. Kybernetika (Prague), 42(2):151-160, 2006.

[50] R. Mesiar, J. Špirková, and L. Vavríková. Weighted aggregation operators based on minimization. Inform. Sci., 178(4):1133-1140, 2008.

[51] A. Mesiarová. Continuous triangular subnorms. Fuzzy Sets and Systems, 142(1):75-83, 2004. Aggregation techniques.

[52] A. Mesiarová. Generators of triangular norms. In Logical, algebraic, analytic, and probabilistic aspects of triangular norms, pages 95-111. Elsevier B. V., Amsterdam, 2005.

[53] P. S. Mostert and A. L. Shield. On the structure of semigroups on a compact manifold with boundary. Ann. of Math., 65:117-143, 1957.

[54] R. Moynihan. Infinite $\tau_{T}$ products of distribution functions. J. Austral. Math. Soc. Ser. A, 26(2):227-240, 1978.

[55] R. B. Nelsen. An introduction to copulas, volume 139 of Lecture Notes in Statistics. Springer-Verlag, New York, 1999. 
[56] Y. Ouyang. On the construction of boundary weak triangular norms through additive generators. Nonlinear Analysis, 66:125-130, 2007.

[57] E. Pap. Null-additive Set Functions, volume 337 of Mathematics and its Applications. Kluwer Academic Publishers Group, Dordrecht, 1995.

[58] E. Pap, editor. Handbook of Measure Theory. Vol. I, II. North-Holland, Amsterdam, 2002.

[59] S. Papavlasopoulos, M. Poulos, N. Korfiatis, and G. Bokos. A non-linear index to evaluate a journal's scientific impact. Inform. Sci., 180(11):21562175,2010 .

[60] B. Schweizer and A. Sklar. Statistical metric spaces. Pacific J. Math., 10:313-334, 1960.

[61] B. Schweizer and A. Sklar. Associative functions and abstract semigroups. Publ. Math. Debrecen, 10:69-81, 1963.

[62] B. Schweizer and A. Sklar. Probabilistic metric spaces. North-Holland Series in Probability and Applied Mathematics. North-Holland Publishing Co., New York, 1983.

[63] M. Sklar. Fonctions de répartition à $n$ dimensions et leurs marges. Publ. Inst. Statist. Univ. Paris, 8:229-231, 1959.

[64] F. Suárez García and P. Gil Álvarez. Two families of fuzzy integrals. Fuzzy Sets and Systems, 18(1):67-81, 1986.

[65] M. Sugeno and T. Murofushi. Pseudo-additive measures and integrals. J. Math. Anal. Appl., 122(1):197-222, 1987.

[66] J. A. Tawn. Bivariate extreme value theory: models and estimation. Biometrika, 75(3):397-415, 1988.

[67] V. Torra and Y. Narukawa. Modeling decisions: Information Fusion and Aggregation Operators. Cognitive Technologies. Springer, 2007.

[68] M. Urbański and J. Wạsowski. Fuzzy arithmetic based on boundary weak T-norms. Int. J. Uncertain. Fuzziness Knowledge-Based Systems, 13(1):27$37,2005$.

[69] Z. Xu. Choquet integrals of weighted intuitionistic fuzzy information. Inform. Sci., 180:5:726-736, 2010.

[70] Z. Xu and Q. L. Da. An overview of operators for aggregating information. Int. J. of Intelligent Systems, 18:953-969, 2003.

[71] R. R. Yager. Aggregation operators and fuzzy systems modeling. Fuzzy Sets and Systems, 67(2):129-145, 1994.

[72] R. R. Yager. Criteria importances in OWA aggregation: an application of fuzzy modeling. In Proc. of the 6th IEEE Int. Conf. on Fuzzy Systems (FUZZ'IEEE'97), pages 1677-1682 vol.3, Barcelona, July 1997. 
[73] R. R. Yager. Fusion of ordinal information using weighted median aggregation. Int. J. Approx. Reason., 18(1-2):35-52, 1998.

[74] R. R. Yager and D. Filev. Essentials of fuzzy modelling and control. J. Wiley \& Sons, New York, 1994.

[75] R. R. Yager and A. Rybalov. Uninorm aggregation operators. Fuzzy Sets and Systems, 80:111-120, 1996.

[76] R. R. Yager and A. Rybalov. Understanding the median as a fusion operator. Int. J. Gen. Systems, 26(3):239-263, 1997.

[77] H. Zimmermann and P. Zysno. Latent connectives in human decision making. Fuzzy Sets and Systems, 4:37-51, 1980. 